UT-KOMABA/98-13

June 1998

\title{
Multi-Body Interactions of D-Particles in Supergravity and Matrix Theory
}

\author{
Yuji OKawa $\mathrm{E}$ and TAMiaki Yoneya \\ Institute of Physics, University of Tokyo, Komaba, Tokyo
}

\begin{abstract}
We present detailed analyses of the 3-body interactions of D-particles from both sides of 11 dimensional supergravity and Matrix theory. In supergravity, we derive a complete expression for the classical bosonic effective action for D-particles including 2-and 3-body interaction terms. In Matrix theory, we compute 1-particle irreducible contributions to the eikonal phase shift in the two-loop approximation. The results precisely agree with the predictions from supergravity and thus provide a strong support to the discrete light-cone interpretation of the Matrix-theory conjecture as a possible nonperturbative definition of M-theory.
\end{abstract}

*e-mail address: okawa@hep1.c.u-tokyo.ac.jp

${ }^{\dagger}$ e-mail address: tam@hep1.c.u-tokyo.ac.jp 


\section{Introduction}

It has recently been hoped that D-branes [1] provide a keystone towards long-sought nonperturbative reformulation of string theory. In particular, the point-like D-branes, namely D-particles, may be the fundamental degrees of freedom from the viewpoint of the so-called M-theory [2] which has been proposed as the non-perturbative correspondent of type IIA string theory. In the low-energy approximation, M-theory is expected to be described by 11 dimensional supergravity. Since the initial proposal of Matrix theory [3] by Banks, Fischler, Shenker and Susskind as a possible realization of the above general conjecture, an impressive amount of evidence concerning its relation with eleven-dimensional supergravity has been accumulated.

However, there has also been reported several results (see, e. g. [4] [5] [0] suggest discrepancies between Matrix theory and supergravity. Among such claims, one of the most serious problems seems to be that of the multi-body interactions [6] of Dparticles. In view of the agreement [8] [9] of the finite $N$ two-loop contribution for the twobody interaction of D-particles with that of supergravity, we should naturally expect that supergravity should be consistent with Matrix theory for 3-body interactions too, since the latter are of the same order of magnitude as the two-body interactions, $N^{3} \frac{v^{6}}{r^{14}}$, with respect to all the external variables involved, the masses or the $N$, the relative velocities and distances between the D-particles. In fact, however, there are important subtleties. The emergence of correct results is not automatic in Matrix theory which is only formulated in the light-cone or infinite-momentum frame. If D-particle is interpreted from the viewpoint of 10 dimensional type IIA string theory, the leading two-body interaction cannot detect the non-linear nature of gravity. Because of the BPS property, it turns out that the selfinteraction of gravitons does not contribute to the two-body force even in the two-loop (and higher) approximation. Therefore, the leading 3-body interaction is the first instance where the self-interaction of gravitons enters. In this sense, the 3-body interaction can be regarded as a crucial testing ground where we can check whether the theory becomes the correct non-linear theory as required by Lorentz invariance, since, as is well known, we can reasonably expect that the consistent Lorentz invariant theories of interacting massless spin two particles necessarily include the Einstein gravity at least in the low-energy regime. 
From the viewpoint of 11D supergravity, on the other hand, the leading 2-body interaction itself involves the self-interaction of gravitons, since a D-particle is assumed to be nothing but a particular Kaluza-Klein mode of eleven-dimensional massless graviton. The Lorentz invariance would therefore require that the self-interaction of gravitons in non Kaluza-Klein modes should be correctly reproduced as well. In other words, the agreement of the 3-body interaction between supergravity and Matrix theory would provide strong evidence for the Lorentz invariance of Matrix theory from both the 10 and 11 dimensional standpoints.

Motivated by these considerations, we decided to carry out a detailed investigation of the 3-body interactions of D-particles from the viewpoints of both 11D supergravity and the discrete light-cone formulation of Matrix theory. The present paper is the first report of our work in this direction. Contrary to the previous claim in [6], the exact agreement between supergravity and Matrix theory is found.

In section 2, we present an analysis of the interactions of D-particles in 11D supergravity in the classical approximation. The goal is to derive the effective action, based on which we can compute the scattering phase shift of D-particles in the eikonal approximation. In section 3 , the computation of the scattering phase shift in the two-loop approximation in Matrix theory is carried out. We present a complete closed formula for 1-particle irreducible contributions to the phase shift. The result is an extension of the previous results reported in ref. [8] [9] for two-body interactions. In section 4, the comparison between supergravity and Matrix theory is made by explicitly computing the eikonal phase shift using the effective action obtained in section 2. Finally, in section 5, we conclude with some remarks. Some of detailed formulas are presented in Appendices.

\section{D-Particle effective action in supergravity}

\subsection{Gravitational fields of D-particles}

We first present a detailed derivation of the effective action for D-particle interactions from the viewpoint of eleven dimensional supergravity. A D-particle (more precisely the transverse-scalar part of a graviton wave packet) which is at rest in the 9 dimensional transverse space is then interpreted as a "pencil of gravitational wave" in 11 dimensional 
space-time whose trajectory is given by $x^{+}=0$ with $N$ units of the Kaluza-Klein momentum $p_{-}=N / R$ in the null 11 th direction $x^{-}$which is compactified to a circle of radius $R$. We can effectively represent the D-particle as a singular exact solution of supergravity corresponding to a gravitational shock wave. Upon the reduction to 10 dimensions, it coincides with the exact (but singular at the origin) BPS solution of 10 dimensional type IIA supergravity, with correct coupling with the dilaton and the 1-form R-R gauge field. Since our aim is the comparison with two-loop calculations in Matrix theory which corresponds to the tree contribution in supergravity, it is sufficient to treat only the purely metric part of supergravity. In this approximation, we can neglect the gravitinos and the 3-form field.

Therefore, we can start from the standard Einstein action in eleven dimensions

$$
\begin{gathered}
S=S_{g}+S_{D}, \\
S_{g}=\frac{1}{2 \kappa_{11}^{2}} \int d^{11} x \sqrt{-g} R
\end{gathered}
$$

by adding the auxiliary action $S_{D}$ which takes into account singular (i.e., $\delta$-function) nature of the D-particle solutions. The basic equation is therefore the Einstein equation in 11 dimensional space-time.

$$
G_{\mu \lambda} \equiv R_{\mu \lambda}-\frac{1}{2} g_{\mu \lambda} R=\kappa_{11}^{2} T_{\mu \lambda}
$$

We use the same convention as ref [9] for the Newton constant $\kappa^{2}=16 \pi^{5} / M^{9}$ with $M$ being the 11 dimensional Planck mass. The energy-momentum tensor for a D-particle can be assumed to be

$$
T^{\mu \lambda}(x)=K \int d s \lambda(s) \frac{d x^{\mu}(s)}{d s} \frac{d x^{\lambda}(s)}{d s} \frac{1}{\sqrt{-g(x(s))}} \delta(x-x(s))
$$

corresponding to the action for a massless point particle

$$
S_{p}=\frac{K}{2} \int d s \lambda(s) g_{\mu \lambda}(x(s)) \frac{d x^{\mu}(s)}{d s} \frac{d x^{\lambda}(s)}{d s}
$$

where $\lambda(s)$ is the Lagrange multiplier imposing the massless constraint

$$
g_{\mu \lambda}(x(s)) \frac{d x^{\mu}(s)}{d s} \frac{d x^{\lambda}(s)}{d s}=0
$$


and $K$ is an arbitrary constant. The trajectory must obey the geodesic equation,

$$
\frac{d^{2} x^{\mu}}{d \tau^{2}}+\Gamma_{\alpha \beta}^{\mu} \frac{d x^{\alpha}}{d \tau} \frac{d x^{\beta}}{d \tau}=0
$$

with $d \tau=\frac{d s}{\lambda(s)}$, corresponding to the conservation of the energy-momentum tensor

$$
D_{\mu} T^{\mu \lambda}=0
$$

which is required by the Bianchi identity for the Einstein tensor $D_{\mu} G^{\mu \nu} \equiv 0$. Since a D-particle is assumed to have a definite (constant) light-like momentum $p_{-}=N / R$ in the $x_{-}$direction, it is appropriate, following ref. [9], to perform the Legendre transformation

$$
\begin{gathered}
S_{p} \rightarrow S_{p}-\int d s p_{-} \frac{d x^{-}}{d s}, \\
p_{-}=K \lambda(s) g_{\mu-} \frac{d x^{\mu}}{d s} .
\end{gathered}
$$

The conventions for the light-like components are $x^{ \pm}=x^{11} \pm t, A \cdot B=\frac{1}{2}\left(A^{+} B^{-}+\right.$ $\left.A^{-} B^{+}\right)+A_{i} B_{i}, 2 A_{-}=A^{+}, 2 A_{+}=A^{-}$. Throughout the present paper, our choice of the time coordinate for D-particles is $\tau=x^{+} / 2$.

The exact solution corresponding to a D-particle with zero-transverse velocity is given by 10

$$
\begin{gathered}
g_{\mu \nu}=\eta_{\mu \nu}+h_{\mu \nu} \\
h_{\mu \nu}=\frac{15}{(2 \pi)^{4}} \kappa_{11}^{2} p_{-} \delta\left(x^{-}\right) \frac{s_{\mu} s_{\nu}}{r^{7}} \\
T^{\mu \nu}=p_{-} \delta\left(x^{-}\right) \delta^{9}\left(x_{\perp}\right) s^{\mu} s^{\nu}
\end{gathered}
$$

where $s^{\mu}$ is the velocity vector $s^{\mu}=\frac{d x^{\mu}}{d \tau}$ of the trajectory

$$
\left(s^{+}, s^{-}, s_{\perp}\right)=2(1,0,0)
$$

and $r$ is the transverse distance from the D-particle source. The exact nature of the solution comes from the properties that only non-vanishing component of the field $h_{\mu \nu}$ is $h_{--}$and $\partial_{+} h_{--}=0$. Then we can check that the Einstein equation reduces to the linearized Laplace equation

$$
-\frac{1}{2} \triangle h_{\mu \lambda}=\kappa_{11}^{2} T_{\mu \lambda}
$$


where $\triangle$ is the Laplacian in the transverse space whose inverse is given as

$$
\left\langle x\left|\triangle^{-1}\right| y\right\rangle=-\frac{15}{2(2 \pi)^{4}|x-y|^{7}} .
$$

Note that both the trace and the divergence of the field (2.12) vanish. The longitudinal momentum is related to the constant $K$ by

$$
p_{-}=K g_{+-} \frac{d x^{+}}{d \tau}=K .
$$

The BPS property explained by the linearized Laplace equation corresponds to the well known fact that there is no force acting between parallel light pencils in General Relativity. Because of this property, we can take an average over the compactified null direction $x^{-}$ of circumference $2 \pi R$ to represent the state of definite $p_{-}$momentum classically. The energy momentum tensor and the gravitational field then take the forms

$$
\begin{aligned}
T^{\mu \nu} & =\frac{N}{2 \pi R^{2}} \delta^{9}\left(x_{\perp}\right) s^{\mu} s^{\nu}, \\
h_{\mu \nu} & =\frac{15}{(2 \pi)^{4}} \kappa_{11}^{2} \frac{N}{2 \pi R^{2}} \frac{s_{\mu} s_{\nu}}{r^{7}} .
\end{aligned}
$$

The equation of the energy-momentum conservation coincides with the ordinary flat space condition

$$
\partial_{\mu} T^{\mu \nu}=0 .
$$

It should also be noted that the gravitational field (2.19) satisfies $h_{\mu \nu} s^{\mu}=0$ which ensures that the self-gravitating effect vanishes for D-particles. This is important for the consistency of the interpretation of the D-particle as a singular limit of the gravitational wave packet in 11 dimensions.

Generalization to D-particles with non-zero velocities in the transverse direction is obvious: The velocity vector (2.14) is replaced by

$$
\left(s^{+}, s^{-}, s_{i}\right)=\left(2,-\frac{1}{2} v^{2}, v_{i}\right)
$$

with $v_{i} \equiv \frac{d x_{i}}{d \tau}$ and the transverse distance is now

$$
r=\left|x_{i}-x_{i}(\tau)\right|^{2}
$$


with $x_{i}(\tau)$ being the trajectory of the D-particle with respect to the transverse coordinates, $x_{i}(\tau)=x_{i}(0)+v_{i} \tau$. Besides these two changes, the transverse $\delta$ function in the expression (2.18) must be replaced by $\delta^{9}\left(x_{i}-x_{i}(\tau)\right)$ which will be denoted by using the same notation $\delta\left(x_{\perp}\right)$ below as in the case of zero-velocity.

Let us now consider the system of many D-particles in which their relative transverse velocities are not zero. In this case, simple superposition of the above solutions does not give exact solutions. However, we can solve the field equations and the equations of motion successively by making the expansion with respect to the gravitational constant $\kappa_{11}^{2}$, or equivalently to the linearized gravitational field, and derive the effective action for the system of many D-particles by expressing the gravitational field in terms of the D-particle coordinates. Note that, because of the Legendre transformation, the action $S_{D}$ for D-particle sources is now

$$
S_{D}=-\sum_{a} \int d \tau p_{a-} \frac{d x_{a}^{-}}{d \tau}
$$

Note that the original particle action $S_{p}$ vanishes owing to the massless constraint (2.6). The index $a$ labels D-particles in the system and $p_{a-}=N_{a} / R$.

\subsection{The second order solution of the Einstein equation}

Our task is now to solve the Einstein equation (2.3) and the D-particle equations of motion (2.7) with the massless constraint (2.6) to the next order beyond the linearized approximation.

Let us begin by collecting some relevant formulas. The Einstein tensor to the second order with respect to $h_{\mu \lambda}$ is given as

$$
\begin{gathered}
G_{\mu \lambda}=G_{\mu \lambda}^{(1)}+G_{\mu \lambda}^{(2)} \\
G_{\mu \lambda}^{(1)}=\frac{1}{2}\left(-\partial^{2} h_{\mu \lambda}-\partial_{\mu} \partial_{\lambda} h_{\nu}^{\nu}+\partial_{\nu} \partial_{\lambda} h_{\mu}^{\nu}+\partial_{\mu} \partial_{\nu} h_{\lambda}^{\nu}\right) \\
-\frac{1}{2} \eta_{\mu \lambda}\left(-\partial^{2} h_{\nu}^{\nu}+\partial_{\alpha} \partial_{\beta} h^{\alpha \beta}\right), \\
G_{\mu \lambda}^{(2)}=-\frac{1}{2} h_{\nu \kappa}\left(\partial^{\nu} \partial_{\lambda} h_{\mu}^{\kappa}+\partial_{\mu} \partial^{\kappa} h_{\lambda}^{\nu}-\partial^{\nu} \partial^{\kappa} h_{\mu \lambda}-\partial_{\mu} \partial_{\lambda} h^{\nu \kappa}\right)
\end{gathered}
$$




$$
\begin{aligned}
& -\frac{1}{2}\left(\partial_{\mu} h_{\alpha \lambda}+\partial_{\lambda} h_{\alpha \mu}\right) \partial_{\nu} h^{\alpha \nu} \\
& +\frac{1}{2} \partial_{\alpha} h_{\mu \lambda} \partial_{\nu} h^{\nu \alpha}+\frac{1}{2} \partial_{\nu} h_{\alpha \lambda} \partial^{\nu} h_{\mu}^{\alpha}-\frac{1}{2} \partial_{\alpha} h_{\lambda}^{\nu} \partial_{\nu} h_{\mu}^{\alpha} \\
& +\frac{1}{4} \partial_{\lambda} h_{\alpha \nu} \partial_{\mu} h^{\alpha \nu}+\frac{1}{4}\left(\partial_{\mu} h_{\lambda}^{\alpha}+\partial_{\lambda} h_{\mu}^{\alpha}-\partial^{\alpha} h_{\mu \lambda}\right) \partial_{\alpha} h_{\nu}^{\nu} \\
& -\frac{1}{2} h_{\mu \lambda}\left(-\partial^{2} h_{\nu}^{\nu}+\partial_{\alpha} \partial_{\beta} h^{\alpha \beta}\right)-\frac{1}{2} \eta_{\mu \lambda} R^{(2)}
\end{aligned}
$$

where

$$
\begin{aligned}
R^{(2)}= & h^{\alpha \beta}\left(\partial^{2} h_{\alpha \beta}+\partial_{\alpha} \partial_{\beta} h_{\nu}^{\nu}-2 \partial_{\gamma} \partial_{\alpha} h_{\beta}^{\gamma}\right) \\
& +\frac{3}{4} \partial_{\beta} h_{\alpha}^{\gamma} \partial^{\beta} h_{\gamma}^{\alpha}-\frac{1}{4} \partial_{\alpha} h_{\mu}^{\mu} \partial^{\alpha} h_{\nu}^{\nu}-\partial_{\beta} h_{\alpha}^{\beta} \partial_{\gamma} h^{\alpha \gamma}-\frac{1}{2} \partial_{\alpha} h_{\beta}^{\gamma} \partial_{\gamma} h^{\alpha \beta}
\end{aligned}
$$

is the $O\left(h^{2}\right)$ part of the scalar curvature. Here and in what follows, the Lorentz indices are contracted with respect to the flat space metric $\eta_{\mu \nu}$, unless otherwise specified. Note that, with this convention, the Einstein equation is

$$
G_{\mu \lambda}^{(1)}+G_{\mu \lambda}^{(2)}=\kappa_{11}^{2}\left(T_{\mu \lambda}+T_{\lambda}^{\nu} h_{\mu \nu}+T_{\mu}^{\nu} h_{\lambda \nu}\right)
$$

The last two terms in the parenthesis of the right hand side arose due to the lowering of the tensor indices from the original definition of the energy-momentum tensor (2.4). The conservation law of the energy-momentum tensor which is necessary for the consistency of the Einstein equation is

$$
\partial_{\mu} T_{\lambda}^{\mu}+\left(\partial_{\mu} h_{\lambda \kappa}-\frac{1}{2} \partial_{\lambda} h_{\mu \kappa}\right) T^{\mu \kappa}=0
$$

to this order. This is equivalent to the equation of motion for the D-particle

$$
\frac{d^{2}}{d \tau^{2}} x^{\alpha}+\frac{d h_{\nu}^{\alpha}}{d \tau} \frac{d x^{\nu}}{d \tau}=\frac{1}{2} \partial^{\alpha} h_{\mu \nu} \frac{d x^{\mu}}{d \tau} \frac{d x^{\nu}}{d \tau} .
$$

It is easy to show that $(2.29)$ is a consequence of the equation of motion (2.30). The converse is also true due to a general theorem given in [11].

Now, for the source action of the D-particles, solving the massless constraint to the second order of the gravitational field, we find

$$
S_{D}=\sum_{a} \frac{N_{a}}{R} \int d \tau\left[\frac{1}{2}\left(\frac{d x_{a}^{i}}{d \tau}\right)^{2}+\frac{1}{2} h_{\mu \nu} s_{a}^{\mu} s_{a}^{\nu}\left(1-h_{-\lambda} s_{a}^{\lambda}\right)\right] .
$$


On the other hand, the structure of the pure gravity action is symbolically of the form

$$
S_{g}=\frac{1}{2 \kappa_{11}^{2}} \int d^{11} x\left(\frac{1}{4} h \mathcal{D} h+\frac{1}{3} V_{3} h h h\right)
$$

to the cubic order where $\mathcal{D}$ and $V_{3}$ denote the differential kinetic operator and the 3-point vertex, respectively. In this notation, the Einstein equation is

$$
\left(-\frac{1}{2} \mathcal{D} h-V_{3} h h\right)^{\mu \nu}=\kappa_{11}^{2} T^{\mu \nu} .
$$

Since our purpose is to derive the effective action for D-particles, we can use the field equation (2.33) inside the action (2.32) and obtain

$$
S_{g}=\frac{1}{2 \kappa_{11}^{2}} \int d^{11} x\left(\frac{1}{12} h \mathcal{D} h-\frac{1}{3} \kappa_{11}^{2} h T\right) .
$$

We denote the energy-momentum tensor and the gravitational field in the linearized approximation using different notations as $\zeta_{\mu \lambda}$ and $\tau_{\mu \lambda}$, respectively. Thus

$$
\begin{gathered}
\zeta_{\mu \lambda}=\sum_{a} \frac{15}{(2 \pi)^{4}} \kappa_{11}^{2} \frac{N_{a}}{2 \pi R^{2}} \frac{s_{a \mu} s_{a \lambda}}{r_{a}^{7}}, \\
\tau_{\mu \lambda}=\sum_{a} \frac{N_{a}}{2 \pi R^{2}} \delta^{9}\left(x_{a \perp}\right) s_{a \mu} s_{a \lambda}
\end{gathered}
$$

which satisfy

$$
-\frac{1}{2} \partial^{2} \zeta_{\mu \lambda}=-\frac{1}{2} \triangle \zeta_{\mu \lambda}=\kappa_{11}^{2} \tau_{\mu \lambda}
$$

and $\partial_{\mu} \zeta^{\mu \lambda}=\zeta_{\mu}^{\mu}=0$. The $\kappa_{11}^{4}$ term of the gravitational field is denoted by $\chi_{\mu \lambda}$ :

$$
h_{\mu \lambda}=\zeta_{\mu \lambda}+\chi_{\mu \lambda} .
$$

We have to compute the total action up to the order $\kappa_{11}^{4}$ so that the gravitational field is of the same order. In order to ensure the consistency of the field equation to this order, the conservation equation (2.29) demands

$$
\partial_{\mu} T_{\lambda}^{\mu}+\left(\partial_{\mu} \zeta_{\lambda \kappa}-\frac{1}{2} \partial_{\lambda} \zeta_{\mu \kappa}\right) \tau^{\mu \kappa}=0
$$

which requires that each trajectory $x_{a}^{\mu}(\tau)$ of D-particles inside the energy-momentum tensor $\tau^{\mu \lambda}$ must be deformed to $x_{a}^{\mu}+\delta x_{a}^{\mu}$ such that the geodesic equations are satisfied. This is nothing but the recoil effect caused by 2-body force. Therefore this effect can be 
neglected for obtaining the genuine 3-body forces. We thus arrive at the equation which determines the second order term $\chi_{\mu \lambda}$ of the gravitational field $h_{\mu \lambda}$ in terms of the field $\zeta_{\mu \lambda}$ of the linear approximation :

$$
G_{\mu \lambda}^{(1)}(\chi)+G_{\mu \lambda}^{(2)}(\zeta)=\kappa_{11}^{2}\left[\tau_{\lambda}^{\nu} \zeta_{\mu \nu}+\tau_{\mu}^{\nu} \zeta_{\lambda \nu}+(\text { recoil })_{\mu \lambda}\right]
$$

where the $\chi$ and $\zeta$ in the left hand side mean that the gravitational field $h$ is now $\chi$ or $\zeta$, respectively, and $(\text { recoil })_{\mu \lambda}$ collectively represents the recoil effect which can be effectively treated as zero in obtaining the effective multi-body forces for D-particles. For the purpose of comparison with Matrix theory calculation in the next section where the recoil effect is completely neglected, this is sufficient. However, it should be kept in mind that taking into account the recoil effect is important for full computation of the action of D-particles.

Then by substituting $h_{\mu \nu}=\zeta_{\mu \nu}+\chi_{\mu \nu}$ in (2.34) and using $(\mathcal{D} \zeta)^{\mu \nu}=-2 \kappa_{11}^{2} \tau^{\mu \nu}$, the gravitational action to the order $\kappa_{11}^{4}$ is given by

$$
S_{g}=-\int d^{11} x\left(\frac{1}{4} \zeta_{\mu \nu} \tau^{\mu \nu}+\frac{1}{3} \chi_{\mu \nu} \tau^{\mu \nu}\right)
$$

This shows that, since the lowest energy-momentum tensor $\tau^{\mu \lambda}$ is traceless, we can safely neglect the trace part of the second order gravitational field $\chi_{\mu \nu}$ within our approximation. Taking into account all these properties, we can write down the equation for the second order gravitational field $\chi$

$$
\begin{aligned}
\frac{1}{2} \partial^{2} \chi_{\mu \lambda}= & -\frac{1}{2} \zeta_{\nu \kappa}\left(\partial^{\nu} \partial_{\lambda} \zeta_{\mu}^{\kappa}+\partial_{\mu} \partial^{\kappa} \zeta_{\lambda}^{\nu}-\partial^{\nu} \partial^{\kappa} \zeta_{\mu \lambda}-\partial_{\mu} \partial_{\lambda} \zeta^{\nu \kappa}\right) \\
& -\frac{1}{2} \partial_{\alpha} \zeta_{\nu \lambda} \partial^{\nu} \zeta_{\mu}^{\alpha}+\frac{1}{4} \partial_{\lambda} \zeta_{\alpha \nu} \partial_{\mu} \zeta^{\alpha \nu} \\
& +\frac{1}{2} \partial_{\nu} \zeta_{\alpha \lambda} \partial^{\nu} \zeta_{\mu}^{\alpha}-\kappa_{11}^{2}\left(\tau_{\lambda}^{\nu} \zeta_{\mu \nu}+\tau_{\mu}^{\nu} \zeta_{\lambda \nu}\right) \\
& +(\text { tracepart })_{\mu \lambda}+(\text { recoil })_{\mu \lambda}
\end{aligned}
$$

where the term (tracepart) ${ }_{\mu \lambda}$, denoting the part proportional to $\eta_{\mu \lambda}$, does not contribute to the effective action, and the term $(\text { recoil })_{\mu \lambda}$ can be neglected for our purpose. Here we have used the fact that the linearized field $\zeta_{\mu \nu}$ has no divergence and trace, and, for $\chi_{\mu \nu}$, have chosen the standard de Donder gauge $\partial^{\mu} \psi_{\mu \nu} \equiv \partial^{\mu}\left(\chi_{\mu \nu}-\frac{1}{2} \eta_{\mu \nu} \chi_{\alpha}^{\alpha}\right)=0$ which leads to $G_{\mu \nu}^{(1)}(\chi)=-\frac{1}{2} \partial^{2} \psi_{\mu \nu}$. This gauge choice is possible, since we have ensured the consistency 
of the Einstein equation in the successive approximation in the expansion with respect to $\kappa_{11}^{2}$. Note that the divergence of the field equation (2.40) leads to the condition on the energy-momentum tensor which is equivalent with the conservation law and hence with the equation of motion. What we have shown above is that an apparent inconsistency which appears if one retains only the terms form the first to the third lines in the equation (2.42) can be attributed to the recoil effect.

Since the trace part does not contribute to the effective action, we can actually neglect the difference between the $\chi_{\mu \nu}$ and $\psi_{\mu \nu}=\chi_{\mu \nu}-\frac{1}{2} \eta_{\mu \nu} \chi_{\alpha}^{\alpha}$. In what follows, for simplicity of the notation, we will always suppress those terms which do not contribute to the final effective action.

Since the field $\zeta_{\mu \nu}$ is independent of $x^{-}$, the equation (2.42) can be inverted using the Laplacian in the transverse space. We call the contributions from the first and the second lines in the right hand side the Y-contribution, and those from the third line the $\mathrm{V}_{g^{-}}$-contribution. Substituting the explicit forms for the linearized fields (2.35) and (2.36), we find that the Y-contribution at the transverse position $x_{a}$ can be written as

$$
\begin{aligned}
\chi_{\mu \lambda}^{\mathrm{Y}}\left(x_{a}\right)= & -\sum_{b, c} \frac{(15)^{3} N_{b} N_{c}}{4(2 \pi)^{4} R^{4} M^{18}}\left[-\frac{1}{2}\left(s_{b} \cdot s_{c}\right)\left(s_{b} \cdot \tilde{\partial}_{c}\right)\left(s_{c \mu} \tilde{\partial}_{c \lambda}+s_{c \lambda} \tilde{\partial}_{c \mu}\right)\right. \\
& +\frac{1}{2}\left(s_{b} \cdot \tilde{\partial}_{c}\right)^{2} s_{c \mu} s_{c \lambda}+\frac{1}{2}\left(s_{b} \cdot s_{c}\right)^{2} \tilde{\partial}_{c \mu} \tilde{\partial}_{c \lambda} \\
& \left.-\frac{1}{2}\left(s_{b} \cdot \tilde{\partial}_{c}\right)\left(s_{c} \cdot \tilde{\partial}_{b}\right) s_{b \lambda} s_{c \mu}+\frac{1}{4}\left(s_{b} \cdot s_{c}\right)^{2} \tilde{\partial}_{b \lambda} \tilde{\partial}_{c \mu}\right] \Delta(a, b, c)
\end{aligned}
$$

where

$$
\begin{gathered}
\Delta(a, b, c) \equiv \int d^{9} y \frac{1}{\left|x_{a}-y\right|^{7}\left|x_{b}-y\right|^{7}\left|x_{c}-y\right|^{7}} \\
=\frac{64(2 \pi)^{3}}{(15)^{3}} \int_{0}^{\infty} d^{3} \sigma\left(\sigma_{1} \sigma_{2}+\sigma_{2} \sigma_{3}+\sigma_{3} \sigma_{1}\right)^{3 / 2} \exp \left(-\sigma_{1}\left|x_{a}-x_{b}\right|^{2}-\sigma_{2}\left|x_{b}-x_{c}\right|^{2}-\sigma_{3}\left|x_{c}-x_{a}\right|^{2}\right)
\end{gathered}
$$

with $d^{3} \sigma=d \sigma_{1} d \sigma_{2} d \sigma_{3}$ and the notation $\tilde{\partial}$ is defined by $\tilde{\partial}_{\mu}=\left(\partial_{+}, 0,-\partial_{i}\right)$.

For the $\mathrm{V}_{g}$-contribution, we use the equality

$$
\triangle^{-1} \partial_{\nu} \zeta_{\alpha \mu} \partial^{\nu} \zeta_{\lambda}^{\alpha}=\frac{1}{2} \zeta_{\alpha \lambda} \zeta_{\mu}^{\alpha}+\kappa_{11}^{2} \triangle^{-1}\left(\tau_{\alpha \lambda} \zeta_{\mu}^{\alpha}+\zeta_{\alpha \lambda} \tau_{\mu}^{\alpha}\right)
$$

for the first term in the third line and, after summing up all terms, obtain

$$
\chi_{\mu \lambda}^{V_{g}}\left(x_{a}\right)=\sum_{b, c} \frac{(15)^{2} N_{b} N_{c}}{8 R^{4} M^{18}}\left(s_{b} \cdot s_{c}\right) s_{b \lambda} s_{c \mu}\left(\frac{1}{r_{a b}^{7}} \frac{1}{r_{a c}^{7}}+\frac{1}{r_{a b}^{7}} \frac{1}{r_{b c}^{7}}+\frac{1}{r_{a c}^{7}} \frac{1}{r_{b c}^{7}}\right)
$$


where $r_{b c}$ is the transverse distance between the D-particles : $r_{b c}=\left|x_{b}-x_{c}\right|$.

\subsection{The effective action of D-particles}

We can now derive the effective action. The contribution from the pure gravity part (2.41) is

$$
S_{g}=-\frac{1}{4} \sum_{a} \frac{N_{a}}{R} \int d \tau \zeta_{a \mu \lambda} s_{a}^{\mu} s_{a}^{\lambda}-\frac{1}{3} \sum_{a} \frac{N_{a}}{R} \int d \tau \chi_{a \mu \lambda} s_{a}^{\mu} s_{a}^{\lambda} .
$$

The source part (2.31) gives

$$
S_{D}=\sum_{a} \frac{N_{a}}{R} \int d \tau\left[\frac{1}{2}\left(\frac{d x_{a}^{i}}{d \tau}\right)^{2}+\frac{1}{2} \zeta_{a \mu \nu} s_{a}^{\mu} s_{a}^{\nu}+\frac{1}{2} \chi_{a \mu \nu} s_{a}^{\mu} s_{a}^{\nu}-\frac{1}{2} \zeta_{a \mu \nu} \zeta_{a-\lambda} s_{a}^{\mu} s_{a}^{\nu} s_{a}^{\lambda}\right] .
$$

Thus the total effective action is

$$
S_{e f f}=\sum_{a} \int d \tau \frac{N_{a}}{R}\left[\frac{1}{2}\left(\frac{d x_{a}^{i}}{d \tau}\right)^{2}+\frac{1}{4} \zeta_{a \mu \nu} s_{a}^{\mu} s_{a}^{\nu}+\frac{1}{6} \chi_{a \mu \nu} s_{a}^{\mu} s_{a}^{\nu}-\frac{1}{2} \zeta_{a \mu \nu} \zeta_{a-\lambda} s_{a}^{\mu} s_{a}^{\nu} s_{a}^{\lambda}\right] .
$$

The second term gives the familiar 2-body lagrangian

$$
L_{2}=\sum_{a<b} \frac{15 N_{a} N_{b}}{16 R^{3} M^{9}} \frac{v_{a b}^{4}}{r_{a b}^{7}} .
$$

We have used the relation $s_{a} \cdot s_{b}=-\frac{1}{2}\left(v_{a}-v_{b}\right)^{2} \equiv-\frac{1}{2} v_{a b}^{2}$ with $v_{a}=\frac{d x_{a}}{d \tau}$. The third and the fourth terms contain the 3-body force. Using (2.43) and (2.46), we express the 3-body term as a sum of two terms

$$
L_{3}=L_{V}+L_{Y}
$$

Note that the V-type contribution consists of two parts corresponding to the contribution of $(2.46)$ and the last term of $(2.49)$

$$
\begin{aligned}
L_{V}= & \sum_{a, b, c} \frac{(15)^{2} N_{a} N_{b} N_{c}}{48 R^{5} M^{18}}\left(s_{b} \cdot s_{c}\right)\left(s_{a} \cdot s_{b}\right)\left(s_{a} \cdot s_{c}\right)\left(\frac{1}{r_{a b}^{7}} \frac{1}{r_{a c}^{7}}+\frac{1}{r_{a b}^{7}} \frac{1}{r_{b c}^{7}}+\frac{1}{r_{a c}^{7}} \frac{1}{r_{b c}^{7}}\right) \\
& -\sum_{a, b, c} \frac{(15)^{2} N_{a} N_{b} N_{c}}{8 R^{5} M^{18}}\left(s_{b} \cdot s_{a}\right)^{2}\left(s_{c} \cdot s_{a}\right) \frac{1}{r_{a b}^{7}} \frac{1}{r_{c a}^{7}} .
\end{aligned}
$$

In terms of the relative velocities, the sum of two terms is rewritten as

$$
L_{V}=-\sum_{a, b, c} \frac{(15)^{2} N_{a} N_{b} N_{c}}{64 R^{5} M^{18}} v_{a b}^{2} v_{c a}^{2}\left(v_{c a} \cdot v_{a b}\right) \frac{1}{r_{a b}^{7}} \frac{1}{r_{c a}^{7}}
$$


The $\mathrm{Y}$ contribution is

$$
\begin{aligned}
L_{Y}= & -\sum_{a, b, c} \frac{(15)^{3} N_{a} N_{b} N_{c}}{24(2 \pi)^{4} R^{5} M^{18}}\left[-\left(s_{b} \cdot s_{c}\right)\left(s_{c} \cdot s_{a}\right)\left(s_{b} \cdot \tilde{\partial}_{c}\right)\left(s_{a} \cdot \tilde{\partial}_{c}\right)\right. \\
& +\frac{1}{2}\left(s_{c} \cdot s_{a}\right)^{2}\left(s_{b} \cdot \tilde{\partial}_{c}\right)^{2}+\frac{1}{2}\left(s_{b} \cdot s_{c}\right)^{2}\left(s_{a} \cdot \tilde{\partial}_{c}\right)^{2} \\
& -\frac{1}{2}\left(s_{b} \cdot s_{a}\right)\left(s_{a} \cdot s_{c}\right)\left(s_{b} \cdot \tilde{\partial}_{c}\right)\left(s_{c} \cdot \tilde{\partial}_{b}\right) \\
& \left.+\frac{1}{4}\left(s_{b} \cdot s_{c}\right)^{2}\left(s_{a} \cdot \tilde{\partial}_{b}\right)\left(s_{a} \cdot \tilde{\partial}_{c}\right)\right] \Delta(a, b, c) .
\end{aligned}
$$

Using $s_{a} \cdot \tilde{\partial}_{c}=\frac{\partial}{\partial \tau_{c}}-v_{a} \cdot \nabla_{c}=v_{c a} \cdot \nabla_{c}$ which is valid because the second derivatives with respect to time can be neglected in the present approximation, we can rewrite this as

$$
\begin{aligned}
L_{Y}= & -\sum_{a, b, c} \frac{(15)^{3} N_{a} N_{b} N_{c}}{96(2 \pi)^{4} R^{5} M^{18}}\left[-v_{b c}^{2} v_{c a}^{2}\left(v_{c b} \cdot \nabla_{c}\right)\left(v_{c a} \cdot \nabla_{c}\right)\right. \\
& +\frac{1}{2} v_{c a}^{4}\left(v_{c b} \cdot \nabla_{c}\right)^{2}+\frac{1}{2} v_{b c}^{4}\left(v_{c a} \cdot \nabla_{c}\right)^{2} \\
& -\frac{1}{2} v_{b a}^{2} v_{a c}^{2}\left(v_{c b} \cdot \nabla_{c}\right)\left(v_{b c} \cdot \nabla_{b}\right) \\
& \left.+\frac{1}{4} v_{b c}^{4}\left(v_{b a} \cdot \nabla_{b}\right)\left(v_{c a} \cdot \nabla_{c}\right)\right] \Delta(a, b, c) .
\end{aligned}
$$

The results (2.53) and (2.55) are our final form of the 3-body interaction Lagrangian of D-particles.

Before proceeding to the investigation of the corresponding matrix model results, it is appropriate here to make some comments on the properties of the above effective Lagrangian.

First, the Y-type interaction (2.55) vanishes when any pair of two D-particles are parallel with vanishing relative velocities. Furthermore, only remaining V-type interaction for such cases is

$$
2 \frac{(15)^{2} N_{a} N_{b} N_{c}}{64 R^{5} M^{18}} v_{a b}^{6} \frac{1}{r_{a b}^{7}} \frac{1}{r_{c a}^{7}}
$$

where the parallel particles are $b$ and $c$. This is just as expected from the BPS property of the D-particles, since the gravitational fields produced by parallel D-particles are exactly the superposition of the linearized fields produced by them.

Secondly, it is remarkable that the kinematical factor of the V-type contributions is reduced to the special combination

$$
v_{a b}^{2} v_{c a}^{2}\left(v_{c a} \cdot v_{a b}\right)=\frac{1}{2} v_{a b}^{2} v_{c a}^{2}\left(v_{b c}^{2}-v_{c a}^{2}-v_{a b}^{2}\right)
$$


From the viewpoint of Matrix theory, this form with the given $r$ dependence is just the typical kinematical factor which arises from a 6-point two-loop diagram of ' $\infty$ ' type in the 'quasi-static' approximation (see, e.g. [12]), with one 4-point vertex $\operatorname{Tr}[A, Y]^{2}$ and three external lines in each loop, entering through the 2-point vertices $\operatorname{Tr}(A[\dot{B}, Y])$, where $A$ and $Y$ are the off-diagonal parts of the gauge field and Higgs fields, respectively. The $\dot{B}$ is the time derivative of the diagonal background $B$ of the Higgs field, corresponding to the velocity of D-particles. This suggests that Matrix theory can be consistent with supergravity, contrary to the claim of ref. [6]. Then the question is whether the other possible types of kinematical factors are canceled and whether the coefficients and also the Y-type contribution come out correctly. In the next section, we will confirm this expectation by carrying out a complete calculation of the two-loop diagrams without relying upon the quasi-static approximation.

Thirdly, it should be noted that in the derivation of the 2-body interaction in ref. 9] only the action for the probe D-particle is taken into account. In contrast with this, in our derivation, the pure gravity action is included. We emphasize that, for the derivation of the effective action for general configurations of D-particles in which all the D-particles are treated democratically as in our formulation, it is essential to include the pure gravity action. In the system of two D-particles, we can always take the Galilei frame such that one of them (source) is at rest in the transverse space. In this case, the total action excluding the 3(and higher)-body interactions can be reduced to the single particle action for the probe since the pure gravity action just cancels the action of the source.

Finally, in our derivation of the effective action we have represented D-particles as the singular solution of the Einstein equation. Correspondingly, we have introduced the source action for D-particles. However, D-particles are supposed to be the special states of the 11 dimensional graviton super multiplet. In this sense, the point-like source variables in the lowest energy-momentum tensor $\tau_{\mu \nu}$ should be interpreted as the collective coordinate of the background gravitational field. From this viewpoint, a conceptually more satisfactory treatment would be to introduce some non-singular D-particle background with finite extension for the metric and take the point-like limit afterwards. In the present paper, however, we do not elaborate along this line since we expect that both treatments lead to the same results. For examples of such treatments in the case of a test point particle in 
General Relativity, see ref. [13] and references therein.

\section{Multi-body D-particle scattering in Matrix theory}

Let us now discuss the multi-body scattering of D-particles or Kaluza-Klein gravitons in Matrix theory up to the two-loop approximation. To make the present paper reasonably self-contained, we start from summarizing some basic facts and formulas of Matrix theory.

\subsection{The setup}

Matrix theory is defined by the action of the supersymmetric Yang-Mills theory dimensionally reduced from $9+1$ dimensions to $0+1$ dimension

$$
\begin{array}{r}
S=\int d t\left[\frac{\kappa}{2} \operatorname{tr} D_{t} X^{n} D_{t} X^{n}+\frac{\kappa}{4} g^{2} \operatorname{tr}\left[X^{n}, X^{m}\right]\left[X^{n}, X^{m}\right]\right. \\
\left.+\frac{\kappa}{2} \operatorname{tr}\left(i \theta^{T} D_{t} \theta+g \theta^{T} \gamma^{n}\left[X^{n}, \theta\right]\right)\right],
\end{array}
$$

where $g$ is the Yang-Mills coupling constant and $n, m=1,2, \cdots, 9$ stand for transverse dimensions. The covariant derivative is defined by

$$
D_{t} X^{n}=\partial_{t} X^{n}-i g\left[A, X^{n}\right], \quad D_{t} \theta=\partial_{t} \theta-i g[A, \theta]
$$

$\gamma^{n}$ are $S O(9)$ gamma matrices in Majorana representation. We take $\gamma^{n}$ to be real and

symmetric matrices satisfying $\left\{\gamma^{n}, \gamma^{m}\right\}=2 \delta^{n m}$ and $\theta$ to be real. $X^{n}, A$ and $\theta$ are hermitian $U(N)$ matrices. For the Grassmann matrix $\theta$, the reality condition is defined by the Majorana condition. The parameters $\kappa$ and $g$ are related to the M-theory parameters as $\kappa=1 / R, g=M^{3} R$.

We will perform our computations in Euclidean formulation, defining the Euclidean time $\tau$ and gauge field in Euclidean time $\tilde{A}$ as

$$
\tau=i t, \quad \tilde{A}=-i A
$$

The time variable $\tau$ of the previous section should be identified with $t$. The Euclidean action is then defined by

$$
\begin{array}{r}
S_{E}=\int d \tau\left[\frac{\kappa}{2} \operatorname{tr} D_{\tau} X^{n} D_{\tau} X^{n}-\frac{\kappa}{4} g^{2} \operatorname{tr}\left[X^{n}, X^{m}\right]\left[X^{n}, X^{m}\right]\right. \\
\left.+\frac{\kappa}{2} \operatorname{tr}\left(\theta^{T} D_{\tau} \theta-g \theta^{T} \gamma^{n}\left[X^{n}, \theta\right]\right)\right],
\end{array}
$$


where the definition of the covariant derivative has changed to

$$
D_{\tau} X^{n}=\partial_{\tau} X^{n}-i g\left[\tilde{A}, X^{n}\right], \quad D_{\tau} \theta=\partial_{\tau} \theta-i g[\tilde{A}, \theta] .
$$

We expand the action around the classical background field $B^{n}$ as

$$
X^{n}=\frac{1}{g} B^{n}+Y^{n} .
$$

We choose the background configuration to be the straight-line trajectories of $N$ Dparticles

$$
B_{i j}^{n}=\delta_{i j}\left(x_{i}^{n}+\tilde{v}_{i}^{n} \tau\right)
$$

where $i, j$ are $U(N)$ indices. Note that the background matrices are actually assumed to consist of several block submatrices which are proportional to the unit matrices and that the order $N_{a}(a=1,2, \ldots)\left(N=\sum_{a} N_{a}\right)$ of each block determines the mass $N_{a} / R$ of the corresponding D-particle. Note also that we have rescaled the background coordinates at time $\tau=0$ and velocities by $x \rightarrow \frac{1}{g} x, v \rightarrow \frac{i}{g} \tilde{v}$. Throughout the present section, the following abbreviations will be used:

$$
\begin{aligned}
& r_{i}^{n}(\tau)=x_{i}^{n}+\tilde{v}_{i}^{n} \tau, \\
& r_{i j}^{n}(\tau)=r_{i}^{n}(\tau)-r_{j}^{n}(\tau), \quad r_{i j}(\tau)^{2}=r_{i j}^{n}(\tau) r_{i j}^{n}(\tau), \quad r_{i j}(\tau)=\sqrt{r_{i j}(\tau)^{2}}, \quad r_{i j}(\tau)=\gamma^{n} r_{i j}^{n}(\tau), \\
& x_{i j}^{n}=x_{i}^{n}-x_{j}^{n}, \quad x_{i j}^{2}=x_{i j}^{n} x_{i j}^{n}, \quad x_{i j}=\sqrt{x_{i j}^{2}}, \quad \not x_{i j}=\gamma^{n} x_{i j}^{n}, \\
& \tilde{v}_{i j}^{n}=\tilde{v}_{i}^{n}-\tilde{v}_{j}^{n}, \quad \tilde{v}_{i j}^{2}=\tilde{v}_{i j}^{n} \tilde{v}_{i j}^{n}, \quad \tilde{v}_{i j}=\sqrt{\tilde{v}_{i j}^{2}}, \quad \ddot{\psi}_{i j}=\gamma^{n} \tilde{v}_{i j}^{n}, \quad x_{i j} \cdot \tilde{v}_{i j}=x_{i j}^{n} \tilde{v}_{i j}^{n} .
\end{aligned}
$$

We use the standard background field gauge condition

$$
-\partial_{\tau} \tilde{A}+i\left[B^{n}, Y^{n}\right]=0,
$$

with the gauge-fixed action

$$
\begin{aligned}
\tilde{S}=\int d & {\left[\frac{\kappa}{g^{2}} \operatorname{tr} \frac{1}{2} \partial_{\tau} B^{n} \partial_{\tau} B^{n}\right.} \\
& +\kappa \operatorname{tr}\left(\frac{1}{2} \partial_{\tau} Y^{n} \partial_{\tau} Y^{n}-\frac{1}{2}\left[B^{m}, Y^{n}\right]\left[B^{m}, Y^{n}\right]+\frac{1}{2} \partial_{\tau} \tilde{A} \partial_{\tau} \tilde{A}-\frac{1}{2}\left[B^{m}, \tilde{A}\right]\left[B^{m}, \tilde{A}\right]\right. \\
& \left.-2 i \partial_{\tau} B^{n}\left[\tilde{A}, Y^{n}\right]-\bar{c} \partial_{\tau}^{2} c+\bar{c}\left[B^{m},\left[B^{m}, c\right]\right]+\frac{1}{2} \theta^{T} \partial_{\tau} \theta-\frac{1}{2} \theta^{T} \gamma^{n}\left[B^{n}, \theta\right]\right)
\end{aligned}
$$




$$
\begin{aligned}
& +g \kappa \operatorname{tr}\left(-i \partial_{\tau} Y^{n}\left[\tilde{A}, Y^{n}\right]-\left[B^{n}, Y^{m}\right]\left[Y^{n}, Y^{m}\right]-\left[B^{n}, \tilde{A}\right]\left[Y^{n}, \tilde{A}\right]\right. \\
& \left.-i \partial_{\tau} \bar{c}[\tilde{A}, c]-\left[B^{n}, \bar{c}\right]\left[Y^{n}, c\right]-\frac{i}{2} \theta^{T}[\tilde{A}, \theta]-\frac{1}{2} \theta^{T} \gamma^{n}\left[Y^{n}, \theta\right]\right) \\
& \left.+g^{2} \kappa \operatorname{tr}\left(-\frac{1}{4}\left[Y^{n}, Y^{m}\right]\left[Y^{n}, Y^{m}\right]-\frac{1}{2}\left[\tilde{A}, Y^{m}\right]\left[\tilde{A}, Y^{m}\right]\right)\right] .
\end{aligned}
$$

Now from the quadratic part of the action

$$
\begin{array}{r}
\tilde{S}_{(2)}=\int d \tau\left[\frac{\kappa}{2}\left(\partial_{\tau} Y_{i j}^{n} \partial_{\tau} Y_{j i}^{n}+r_{i j}(\tau)^{2} Y_{i j}^{n} Y_{j i}^{n}\right)+\frac{\kappa}{2}\left(\partial_{\tau} \tilde{A}_{i j} \partial_{\tau} \tilde{A}_{j i}+r_{i j}(\tau)^{2} \tilde{A}_{i j} \tilde{A}_{j i}\right)\right. \\
-2 i \kappa \tilde{v}_{i j}^{n} \tilde{A}_{i j} Y_{j i}^{n}+\kappa\left(-\bar{c}_{i j} \partial_{\tau}^{2} c_{j i}+r_{i j}(\tau)^{2} \bar{c}_{i j} c_{j i}\right) \\
\left.+\frac{\kappa}{2}\left(\theta_{i j}^{T} \partial_{\tau} \theta_{j i}+\theta_{i j}^{T} \gamma_{i j}(\tau) \theta_{j i}\right)\right],
\end{array}
$$

we derive the propagators in proper-time representations.

Propagators of the bosonic fields:

$$
\begin{aligned}
\left\langle Y_{i j}^{n}\left(\tau_{1}\right) Y_{k l}^{m}\left(\tau_{2}\right)\right\rangle_{0} & =\frac{\delta_{i l} \delta_{j k}}{\kappa} \int_{0}^{\infty} d \sigma\left(\delta^{n m}+2 V_{i j}^{n}(\sigma) V_{i j}^{m}(\sigma)\right) \Delta_{i j}\left(\sigma, \tau_{1}, \tau_{2}\right), \\
\left\langle\tilde{A}_{i j}\left(\tau_{1}\right) \tilde{A}_{k l}\left(\tau_{2}\right)\right\rangle_{0} & =\frac{\delta_{i l} \delta_{j k}}{\kappa} \int_{0}^{\infty} d \sigma\left(1+2 V_{i j}(\sigma)^{2}\right) \Delta_{i j}\left(\sigma, \tau_{1}, \tau_{2}\right), \\
\left\langle Y_{i j}^{n}\left(\tau_{1}\right) \tilde{A}_{k l}\left(\tau_{2}\right)\right\rangle_{0} & =\frac{2 i \delta_{i l} \delta_{j k}}{\kappa} \int_{0}^{\infty} d \sigma V_{i j}^{n}(\sigma) C_{i j}(\sigma) \Delta_{i j}\left(\sigma, \tau_{1}, \tau_{2}\right), \\
\left\langle\tilde{A}_{i j}\left(\tau_{1}\right) Y_{k l}^{m}\left(\tau_{2}\right)\right\rangle_{0} & =-\frac{2 i \delta_{i l} \delta_{j k}}{\kappa} \int_{0}^{\infty} d \sigma V_{i j}^{m}(\sigma) C_{i j}(\sigma) \Delta_{i j}\left(\sigma, \tau_{1}, \tau_{2}\right), \\
\left\langle\bar{c}_{i j}\left(\tau_{1}\right) c_{k l}\left(\tau_{2}\right)\right\rangle_{0} & =\frac{\delta_{i l} \delta_{j k}}{\kappa} \int_{0}^{\infty} d \sigma \Delta_{i j}\left(\sigma, \tau_{1}, \tau_{2}\right), \\
\left\langle c_{i j}\left(\tau_{1}\right) \bar{c}_{k l}\left(\tau_{2}\right)\right\rangle_{0} & =-\frac{\delta_{i l} \delta_{j k}}{\kappa} \int_{0}^{\infty} d \sigma \Delta_{i j}\left(\sigma, \tau_{1}, \tau_{2}\right) .
\end{aligned}
$$

Propagators of the fermionic fields:

$$
\begin{aligned}
& \left\langle\theta_{i j}^{\alpha}\left(\tau_{1}\right) \theta_{k l}^{\beta}\left(\tau_{2}\right)\right\rangle_{0} \\
& =\frac{\delta_{i l} \delta_{j k}}{\kappa} \int_{0}^{\infty} d \sigma[- \\
& \left.\quad\left(1 C_{i j}(\sigma) \partial_{\tau_{1}}+\nvdash_{i j}\left(\tau_{1}\right) V_{i j}(\sigma)\right)-\left(V_{i j}(\sigma) \partial_{\tau_{1}}+C_{i j}(\sigma) \gamma_{i j}\left(\tau_{1}\right)\right)\right]^{\alpha \beta} \\
& \times \Delta_{i j}\left(\sigma, \tau_{1}, \tau_{2}\right)
\end{aligned}
$$

or

$$
\begin{aligned}
& \left\langle\theta_{k l}^{\beta}\left(\tau_{2}\right) \theta_{i j}^{\alpha}\left(\tau_{1}\right)\right\rangle_{0} \\
& =\frac{\delta_{i l} \delta_{j k}}{\kappa} \int_{0}^{\infty} d \sigma[- \\
& \left.\quad\left(\mathbf{1} C_{i j}(\sigma) \partial_{\tau_{2}}+\gamma_{i j}\left(\tau_{2}\right) V_{i j}(\sigma)\right)+\left(V_{i j}(\sigma) \partial_{\tau_{2}}+C_{i j}(\sigma) \gamma_{i j}\left(\tau_{2}\right)\right)\right]^{\beta \alpha} \\
& \times \Delta_{i j}\left(\sigma, \tau_{1}, \tau_{2}\right) .
\end{aligned}
$$


Here we have defined

$$
\begin{aligned}
& \Delta_{i j}\left(\sigma, \tau_{1}, \tau_{2}\right) \equiv \exp \left[-\sigma\left(-\partial_{\tau_{1}}^{2}+r^{2}\left(\tau_{1}\right)\right)\right] \delta\left(\tau_{1}-\tau_{2}\right) \\
&= \sqrt{\frac{\tilde{v}_{i j}}{2 \pi \sinh \left(2 \sigma \tilde{v}_{i j}\right)}} \exp \left[-\frac{\tilde{v}_{i j}}{2 \sinh \left(2 \sigma \tilde{v}_{i j}\right)}\left(\left(\tau_{1}^{2}+\tau_{2}^{2}\right) \cosh \left(2 \sigma \tilde{v}_{i j}\right)-2 \tau_{1} \tau_{2}\right)\right. \\
&\left.-\frac{x_{i j} \cdot \tilde{v}_{i j} \tanh \left(\sigma \tilde{v}_{i j}\right)}{\tilde{v}_{i j}}\left(\tau_{1}+\tau_{2}+\frac{x_{i j} \cdot \tilde{v}_{i j}}{\tilde{v}_{i j}^{2}}\right)-\sigma\left(x_{i j}^{2}-\frac{\left(x_{i j} \cdot \tilde{v}_{i j}\right)^{2}}{\tilde{v}_{i j}^{2}}\right)\right] \\
&= \quad \sqrt{\frac{\tilde{v}_{i j}}{2 \pi \sinh \left(2 \sigma \tilde{v}_{i j}\right)}} \exp \left[-\tilde{v}_{i j}\left(\frac{\tau_{1}-\tau_{2}}{2}\right)^{2} \operatorname{coth}\left(\sigma \tilde{v}_{i j}\right)\right. \\
&\left.-\tilde{v}_{i j}\left(\frac{\tau_{1}+\tau_{2}}{2}+\frac{x_{i j} \cdot \tilde{v}_{i j}}{\tilde{v}_{i j}^{2}}\right)^{2} \tanh \left(\sigma \tilde{v}_{i j}\right)-\sigma\left(x_{i j}^{2}-\frac{\left(x_{i j} \cdot \tilde{v}_{i j}\right)^{2}}{\tilde{v}_{i j}^{2}}\right)\right] \\
& V_{i j}^{n}(\sigma) \equiv \frac{\tilde{v}_{i j}^{n}}{\tilde{v}_{i j}} \sinh \left(\sigma \tilde{v}_{i j}\right), \\
&\left.C_{i j}(\sigma) \equiv \tilde{c o s h}_{i j}\right), \\
& V_{i j}(\sigma)^{2} \equiv \tilde{v}_{i j}(\sigma) \cdot V_{i j}(\sigma)=V_{i j}^{n}(\sigma) V_{i j}^{n}(\sigma)=\sinh ^{2}\left(\sigma \tilde{v}_{i j}\right), \\
&\left.V_{i j}(\sigma) \equiv \sinh _{i j}(\sigma) \cdot V_{i j}(\sigma)=\sqrt{V_{i j}^{n}(\sigma) V_{i j}^{n}(\sigma)}=\sin _{i j}\right)
\end{aligned}
$$

\subsection{The calculations of the effective action}

We now present the complete form of the effective action $\tilde{\Gamma}$ around the background configuration in the two-loop approximation

$$
\tilde{\Gamma}=\tilde{\Gamma}_{(0)}+\tilde{\Gamma}_{(1)}+\tilde{\Gamma}_{(2)}+O\left(g^{4}\right)
$$

where $\tilde{\Gamma}_{(\ell)}$ stands for the $\ell$-loop contribution.

We note that the effective action from the viewpoint of the Yang-Mills field theory is nothing but the one-particle irreducible contribution to the eikonal phase shift of Dparticle, since the results contains the time integration of the genuine light-cone lagrangian for straight line trajectories. First, the classical action $\tilde{\Gamma}_{(0)}$ is

$$
\tilde{\Gamma}_{(0)}=\int_{-\infty}^{\infty} d \tau \frac{\kappa}{g^{2}} \operatorname{tr} \frac{1}{2} \partial_{\tau} B^{n} \partial_{\tau} B^{n}=\int_{-\infty}^{\infty} d \tau \frac{1}{R g^{2}} \sum_{i} \frac{1}{2} \tilde{v}_{i}^{2}=\int_{-\infty}^{\infty} d \tau \sum_{a} \frac{N_{a}}{R g^{2}} \tilde{v}_{a}^{2}
$$

where in the last equality, we switched from the summation over the $\mathrm{U}(N)$ indices to the block indices specifying the system of coincident D-particles. 
The one-loop contribution is obtained from the functional determinant as

$$
\begin{aligned}
& \exp \left[-\tilde{\Gamma}_{(1)}\right]=\int D Y^{n} D \tilde{A} D \bar{c} D c D \theta e^{-\tilde{S}_{(2)}} \\
& =\prod_{i<j} \operatorname{det}^{-6}\left(-\partial_{\tau}^{2}+r_{i j}(\tau)^{2}\right) \operatorname{det}^{-1}\left(-\partial_{\tau}^{2}+r_{i j}(\tau)^{2}+2 \tilde{v}_{i j}\right) \operatorname{det}^{-1}\left(-\partial_{\tau}^{2}+r_{i j}(\tau)^{2}-2 \tilde{v}_{i j}\right) \\
& \times \operatorname{det}^{4}\left(-\partial_{\tau}^{2}+r_{i j}(\tau)^{2}+\tilde{v}_{i j}\right) \operatorname{det}^{4}\left(-\partial_{\tau}^{2}+r_{i j}(\tau)^{2}-\tilde{v}_{i j}\right) .
\end{aligned}
$$

$\tilde{\Gamma}_{(1)}$ is expressed in the proper-time representation as

$$
\begin{aligned}
\tilde{\Gamma}_{(1)} & =-\sum_{i<j} \int_{-\infty}^{\infty} d \tau \int_{0}^{\infty} \frac{d \sigma}{\sigma} 16 \sinh ^{4} \frac{\sigma \tilde{v}_{i j}}{2} \Delta_{i j}(\sigma, \tau, \tau) \\
& =-\sum_{i<j} \int_{0}^{\infty} \frac{d \sigma}{\sigma} 16 \sinh ^{4} \frac{\sigma \tilde{v}_{i j}}{2} \frac{1}{2 \sinh \left(\sigma \tilde{v}_{i j}\right)} \exp \left[-\sigma\left(x_{i j}^{2}-\frac{\left(x_{i j} \cdot \tilde{v}_{i j}\right)^{2}}{\tilde{v}_{i j}^{2}}\right)\right] .
\end{aligned}
$$

Note that we have slightly generalized the well-known result of the 2-body scattering because we cannot, in general, assume vanishing of $x_{i j} \cdot \tilde{v}_{i j}$ in multi-body scattering. The leading contribution of the one-loop effective action $\tilde{\Gamma}_{(1)}$ is

$$
\begin{aligned}
\tilde{\Gamma}_{(1) \text { leading }} & =-\sum_{i<j} \int_{-\infty}^{\infty} d \tau \int_{0}^{\infty} \frac{d \sigma}{\sigma} \frac{\sigma^{4} \tilde{v}_{i j}^{4}}{\sqrt{4 \pi \sigma}} \exp \left[-\sigma\left(\tilde{v}_{i j} \tau+x_{i j}\right)^{2}\right] \\
& =-\int_{-\infty}^{\infty} d \tau \sum_{i<j} \frac{15}{16} \frac{\tilde{v}_{i j}^{4}}{\left[\left(\tilde{v}_{i j} \tau+x_{i j}\right)^{2}\right]^{7 / 2}}
\end{aligned}
$$

where we used the proper-time representation

$$
\frac{1}{\left[(\tilde{v} \tau+x)^{2}\right]^{7 / 2}}=\frac{8}{15 \sqrt{\pi}} \int_{0}^{\infty} d \sigma \frac{\sigma^{3}}{\sqrt{\sigma}} \exp \left[-\sigma(\tilde{v} \tau+x)^{2}\right] .
$$

Since the one-loop contribution has been studied in many works, no further discussion is needed here.

Two loop calculations have been performed in ref. [8] [9]. In what follows, we will report an extension of their results in order to make possible the comparison of the 3-body interaction of D-particles with the results of the preceding section. To obtain the two-loop contribution $\tilde{\Gamma}_{(2)}$ to the effective action, we have to evaluate all one-particle irreducible (1PI) diagrams. Since the nonplanar 1PI diagrams at two-loop order have only one index loop and hence do not contribute to D-particle interactions, we can restrict our 
consideration to planar diagrams. There are fourteen different contributions depending on the combinations of the propagating fields:

$$
\begin{aligned}
& -\tilde{\Gamma}_{(2)} \\
& =\frac{1}{4} g^{2} \kappa \int d \tau\left\langle\operatorname{tr}\left[Y^{n}(\tau), Y^{m}(\tau)\right]\left[Y^{n}(\tau), Y^{m}(\tau)\right]\right\rangle_{1 P I, \text { planar }} \\
& +\frac{1}{2} g^{2} \kappa \int d \tau\left\langle\operatorname{tr}\left[\tilde{A}(\tau), Y^{m}(\tau)\right]\left[\tilde{A}(\tau), Y^{m}(\tau)\right]\right\rangle_{1 P I, \text { planar }} \\
& -\frac{1}{2} g^{2} \kappa^{2} \int d \tau_{1} d \tau_{2}\left\langle\operatorname{tr} \partial_{\tau_{1}} Y^{p}\left(\tau_{1}\right)\left[\tilde{A}\left(\tau_{1}\right), Y^{p}\left(\tau_{1}\right)\right] \operatorname{tr} \partial_{\tau_{2}} Y^{q}\left(\tau_{2}\right)\left[\tilde{A}\left(\tau_{2}\right), Y^{q}\left(\tau_{2}\right)\right]\right\rangle_{1 P I, \text { planar }} \\
& +\frac{1}{2} g^{2} \kappa^{2} \int d \tau_{1} d \tau_{2}\left\langle\operatorname{tr}\left[B^{n}, Y^{p}\right]\left(\tau_{1}\right)\left[Y^{n}\left(\tau_{1}\right), Y^{p}\left(\tau_{1}\right)\right] \operatorname{tr}\left[B^{m}, Y^{q}\right]\left(\tau_{2}\right)\left[Y^{m}\left(\tau_{2}\right), Y^{q}\left(\tau_{2}\right)\right]\right\rangle_{1 P I, \text { planar }} \\
& +\frac{1}{2} g^{2} \kappa^{2} \int d \tau_{1} d \tau_{2}\left\langle\operatorname{tr}\left[B^{n}, \tilde{A}\right]\left(\tau_{1}\right)\left[Y^{n}\left(\tau_{1}\right), \tilde{A}\left(\tau_{1}\right)\right] \operatorname{tr}\left[B^{m}, \tilde{A}\right]\left(\tau_{2}\right)\left[Y^{m}\left(\tau_{2}\right), \tilde{A}\left(\tau_{2}\right)\right]\right\rangle{ }_{1 P I, \text { planar }} \\
& +i g^{2} \kappa^{2} \int d \tau_{1} d \tau_{2}\left\langle\operatorname{tr} \partial_{\tau_{1}} Y^{p}\left(\tau_{1}\right)\left[\tilde{A}\left(\tau_{1}\right), Y^{p}\left(\tau_{1}\right)\right] \operatorname{tr}\left[B^{m}, Y^{q}\right]\left(\tau_{2}\right)\left[Y^{m}\left(\tau_{2}\right), Y^{q}\left(\tau_{2}\right)\right]\right\rangle_{1 P I, \text { planar }} \\
& +i g^{2} \kappa^{2} \int d \tau_{1} d \tau_{2}\left\langle\operatorname{tr} \partial_{\tau_{1}} Y^{p}\left(\tau_{1}\right)\left[\tilde{A}\left(\tau_{1}\right), Y^{p}\left(\tau_{1}\right)\right] \operatorname{tr}\left[B^{m}, \tilde{A}\right]\left(\tau_{2}\right)\left[Y^{m}\left(\tau_{2}\right), \tilde{A}\left(\tau_{2}\right)\right]\right\rangle_{1 P I, \text { planar }} \\
& +g^{2} \kappa^{2} \int d \tau_{1} d \tau_{2}\left\langle\operatorname{tr}\left[B^{n}, Y^{p}\right]\left(\tau_{1}\right)\left[Y^{n}\left(\tau_{1}\right), Y^{p}\left(\tau_{1}\right)\right] \operatorname{tr}\left[B^{m}, \tilde{A}\right]\left(\tau_{2}\right)\left[Y^{m}\left(\tau_{2}\right), \tilde{A}\left(\tau_{2}\right)\right]\right\rangle_{1 P I, \text { planar }} \\
& -\frac{1}{2} g^{2} \kappa^{2} \int d \tau_{1} d \tau_{2}\left\langle\operatorname{tr} \partial_{\tau_{1}} \bar{c}\left(\tau_{1}\right)\left[\tilde{A}\left(\tau_{1}\right), c\left(\tau_{1}\right)\right] \operatorname{tr} \partial_{\tau_{2}} \bar{c}\left(\tau_{2}\right)\left[\tilde{A}\left(\tau_{2}\right), c\left(\tau_{2}\right)\right]\right\rangle_{1 P I, \text { planar }} \\
& +\frac{1}{2} g^{2} \kappa^{2} \int d \tau_{1} d \tau_{2}\left\langle\operatorname{tr}\left[B^{n}, \bar{c}\right]\left(\tau_{1}\right)\left[Y^{n}\left(\tau_{1}\right), c\left(\tau_{1}\right)\right] \operatorname{tr}\left[B^{m}, \bar{c}\right]\left(\tau_{2}\right)\left[Y^{m}\left(\tau_{2}\right), c\left(\tau_{2}\right)\right]\right\rangle_{1 P I, \text { planar }} \\
& +i g^{2} \kappa^{2} \int d \tau_{1} d \tau_{2}\left\langle\operatorname{tr} \partial_{\tau_{1}} \bar{c}\left(\tau_{1}\right)\left[\tilde{A}\left(\tau_{1}\right), c\left(\tau_{1}\right)\right] \operatorname{tr}\left[B^{m}, \bar{c}\right]\left(\tau_{2}\right)\left[Y^{m}\left(\tau_{2}\right), c\left(\tau_{2}\right)\right]\right\rangle_{1 P I, \text { planar }} \\
& -\frac{1}{8} g^{2} \kappa^{2} \int d \tau_{1} d \tau_{2}\left\langle\operatorname{tr} \theta^{\alpha}\left(\tau_{1}\right)\left[\tilde{A}\left(\tau_{1}\right), \theta^{\alpha}\left(\tau_{1}\right)\right] \operatorname{tr} \theta^{\beta}\left(\tau_{2}\right)\left[\tilde{A}\left(\tau_{2}\right), \theta^{\beta}\left(\tau_{2}\right)\right]\right\rangle_{1 P I, \text { planar }} \\
& +\frac{1}{8} g^{2} \kappa^{2} \gamma_{\alpha \beta}^{n} \gamma_{\gamma \delta}^{m} \int d \tau_{1} d \tau_{2}\left\langle\operatorname{tr} \theta^{\alpha}\left(\tau_{1}\right)\left[Y^{n}\left(\tau_{1}\right), \theta^{\beta}\left(\tau_{1}\right)\right] \operatorname{tr} \theta^{\gamma}\left(\tau_{2}\right)\left[Y^{m}\left(\tau_{2}\right), \theta^{\delta}\left(\tau_{2}\right)\right]\right\rangle_{1 P I, \text { planar }} \\
& +\frac{i}{4} g^{2} \kappa^{2} \gamma_{\beta \gamma}^{n} \int d \tau_{1} d \tau_{2}\left\langle\operatorname{tr} \theta^{\alpha}\left(\tau_{1}\right)\left[\tilde{A}\left(\tau_{1}\right), \theta^{\alpha}\left(\tau_{1}\right)\right] \operatorname{tr} \theta^{\beta}\left(\tau_{2}\right)\left[Y^{n}\left(\tau_{2}\right), \theta^{\gamma}\left(\tau_{2}\right)\right]\right\rangle_{1 P I, \text { planar }} .
\end{aligned}
$$

Evaluating these terms is a straightforward but extremely tedious task. After substituting the explicit forms of the propagators in the proper time representation, we can arrange the result in the following way:

$$
\begin{aligned}
-\tilde{\Gamma}_{(2)}= & \frac{g^{2}}{\kappa} \int d \tau_{1} d \tau_{2} d \sigma_{1} d \sigma_{2} d \sigma_{3} \sum_{i, j, k}[ \\
& P_{q}(\{\sigma\},\{\tilde{v}\})\left\{-\partial_{\sigma_{1}} \Delta_{i j}\left(\sigma_{1}, \tau_{1}, \tau_{2}\right)\right\} \Delta_{j k}\left(\sigma_{2}, \tau_{1}, \tau_{2}\right) \Delta_{k i}\left(\sigma_{3}, \tau_{1}, \tau_{2}\right) \\
& +P_{1}(\{\sigma\},\{\tilde{v}\})\left\{\partial_{\tau_{1}} \partial_{\tau_{2}} \Delta_{i j}\left(\sigma_{1}, \tau_{1}, \tau_{2}\right)\right\} \Delta_{j k}\left(\sigma_{2}, \tau_{1}, \tau_{2}\right) \Delta_{k i}\left(\sigma_{3}, \tau_{1}, \tau_{2}\right)
\end{aligned}
$$




$$
\begin{aligned}
& +P_{2}\left(\{\sigma\},\{\tilde{v}\},\left\{\tilde{v} \cdot r\left(\tau_{2}\right)\right\}\right)\left\{\partial_{\tau_{1}} \Delta_{i j}\left(\sigma_{1}, \tau_{1}, \tau_{2}\right)\right\} \Delta_{j k}\left(\sigma_{2}, \tau_{1}, \tau_{2}\right) \Delta_{k i}\left(\sigma_{3}, \tau_{1}, \tau_{2}\right) \\
& +P_{3}(\{\sigma\},\{\tilde{v}\})\left(r_{i j}\left(\tau_{1}\right) \cdot r_{i j}\left(\tau_{2}\right)\right) \Delta_{i j}\left(\sigma_{1}, \tau_{1}, \tau_{2}\right) \Delta_{j k}\left(\sigma_{2}, \tau_{1}, \tau_{2}\right) \Delta_{k i}\left(\sigma_{3}, \tau_{1}, \tau_{2}\right) \\
& \left.+P_{4}\left(\{\sigma\},\{\tilde{v}\},\left\{\tilde{v} \cdot r\left(\tau_{1}\right)\right\},\left\{\tilde{v} \cdot r\left(\tau_{2}\right)\right\}\right) \Delta_{i j}\left(\sigma_{1}, \tau_{1}, \tau_{2}\right) \Delta_{j k}\left(\sigma_{2}, \tau_{1}, \tau_{2}\right) \Delta_{k i}\left(\sigma_{3}, \tau_{1}, \tau_{2}\right)\right],
\end{aligned}
$$

where $P$ 's are polynomials with respect to their arguments and $\{\sigma\},\{\tilde{v}\},\{\tilde{v} \cdot r(\tau)\}$ denote the dependence collectively

$$
\begin{aligned}
\{\sigma\} & =\left\{\sigma_{a} \mid a \in\{1,2,3\}\right\} \\
\{\tilde{v}\} & =\left\{\tilde{v}_{a} \mid a \in\{i j, j k, k i\}\right\}, \\
\{\tilde{v} \cdot r(\tau)\} & =\left\{\tilde{v}_{a} \cdot r_{b}(\tau) \mid a, b \in\{i j, j k, k i\}\right\} .
\end{aligned}
$$

The $P_{q}$ term contains the whole of the diagrams (' $\infty$ '-type diagrams) with 4 -point vertices. The appearance of three propagators in it is due to the following transformation:

$$
\begin{aligned}
& \int d \tau d \sigma_{2} d \sigma_{3} \sum_{i, j, k} \Delta_{j k}\left(\sigma_{2}, \tau, \tau\right) \Delta_{k i}\left(\sigma_{3}, \tau, \tau\right) \\
= & \int d \tau_{1} d \tau_{2} d \sigma_{2} d \sigma_{3} \sum_{i, j, k} \delta\left(\tau_{1}-\tau_{2}\right) \Delta_{j k}\left(\sigma_{2}, \tau_{1}, \tau_{2}\right) \Delta_{k i}\left(\sigma_{3}, \tau_{1}, \tau_{2}\right) \\
= & \int d \tau_{1} d \tau_{2} d \sigma_{1} d \sigma_{2} d \sigma_{3} \sum_{i, j, k}\left\{-\partial_{\sigma_{1}} \Delta_{i j}\left(\sigma_{1}, \tau_{1}, \tau_{2}\right)\right\} \Delta_{j k}\left(\sigma_{2}, \tau_{1}, \tau_{2}\right) \Delta_{k i}\left(\sigma_{3}, \tau_{1}, \tau_{2}\right) .
\end{aligned}
$$

The other terms represent the contributions of the diagrams (' $\phi$ '-type diagrams) with two 3 -point vertices. The $P_{1}$ term contains terms with two derivatives operating propertime propagators, and the $P_{2}$ term contains terms with one derivative. The $P_{3}$ and $P_{4}$ terms contain terms which do not involve derivatives. Note that we explicitly separated the terms containing $r_{i j}\left(\tau_{1}\right) \cdot r_{i j}\left(\tau_{2}\right)$ as the $P_{3}$ term from the rest of the contributions which we called the $P_{4}$ term. In deriving the above form, we repeatedly performed partial integrations with respect to $\tau_{1}$ or $\tau_{2}$, using the relation

$$
r_{i j}^{n}(\tau)+r_{j k}^{n}(\tau)+r_{k i}^{n}(\tau)=0
$$

and also redefining the integration variables, $\sigma_{1}, \sigma_{2}, \sigma_{3}, \tau_{1}$ and $\tau_{2}$, and indices $i, j$ and $k$ such as

$$
i \rightarrow j, \quad j \rightarrow k, \quad k \rightarrow i, \quad \sigma_{1} \rightarrow \sigma_{2}, \quad \sigma_{2} \rightarrow \sigma_{3}, \quad \sigma_{3} \rightarrow \sigma_{1},
$$


or

$$
i \rightarrow j, \quad j \rightarrow i, \quad \sigma_{2} \rightarrow \sigma_{3}, \quad \sigma_{3} \rightarrow \sigma_{2}
$$

We will present the explicit forms of $P_{q}, P_{1}, P_{2}, P_{3}$ and $P_{4}$ and some examples of the evaluations of contributions from individual terms in (3.32) in Appendix A and B, respectively.

Let us first focus on how $\tilde{\Gamma}_{(2)}$ depends on $x_{i}^{n}$. There are two types of dependence:

1. $\left\{x^{2}\right\} \equiv\left\{x_{a}^{2} \mid a \in\{i j, j k, k i\}\right\}$,

2. $\{x \cdot \tilde{v}\} \equiv\left\{x_{a} \cdot \tilde{v}_{b} \mid a, b \in\{i j, j k, k i\}\right\}$.

The dependence of the type $\left\{x^{2}\right\}$ exists only in the form $r_{i j}\left(\tau_{1}\right) \cdot r_{i j}\left(\tau_{2}\right)$ except inside the proper-time propagators. The derivatives with respect to $\tau_{1}$ or $\tau_{2}$ acting on proper-time propagators do not induce $\left\{x^{2}\right\}$ dependence as can be seen from the form of the propertime propagator $(3.20)$. The terms with lower powers of $\{\tilde{v}\}$ must cancel in order to agree with the result from the supergravity which shows that the 3-body Lagrangian starts from the order $O\left(\tilde{v}^{6}\right)$. However, it may seem that the cancellations cannot be expected before the proper-time integrations because of the dependence of the type $\left\{x^{2}\right\}$ in $r_{i j}\left(\tau_{1}\right) \cdot r_{i j}\left(\tau_{2}\right)$. In fact, however, using the equations

$$
\begin{aligned}
& {\left[\partial_{\sigma_{1}}-\partial_{\tau_{1}}^{2}+r_{i j}\left(\tau_{1}\right)^{2}\right] \Delta_{i j}\left(\sigma_{1}, \tau_{1}, \tau_{2}\right)=0,} \\
& {\left[\partial_{\sigma_{1}}-\partial_{\tau_{2}}^{2}+r_{i j}\left(\tau_{2}\right)^{2}\right] \Delta_{i j}\left(\sigma_{1}, \tau_{1}, \tau_{2}\right)=0,}
\end{aligned}
$$

we can eliminate the factor $r_{i j}\left(\tau_{1}\right) \cdot r_{i j}\left(\tau_{2}\right)$ as

$$
\begin{aligned}
& \left(r_{i j}\left(\tau_{1}\right) \cdot r_{i j}\left(\tau_{2}\right)\right) \Delta_{i j}\left(\sigma_{1}, \tau_{1}, \tau_{2}\right) \\
& =\left[r_{i j}\left(\tau_{1}\right) \cdot r_{i j}\left(\tau_{2}\right)-\partial_{\sigma_{1}}+\frac{1}{2}\left(\partial_{\tau_{1}}^{2}+\partial_{\tau_{2}}^{2}\right)-\frac{1}{2}\left(r_{i j}\left(\tau_{1}\right)^{2}+r_{i j}\left(\tau_{2}\right)^{2}\right)\right] \Delta_{i j}\left(\sigma_{1}, \tau_{1}, \tau_{2}\right) \\
& =-\partial_{\sigma_{1}} \Delta_{i j}\left(\sigma_{1}, \tau_{1}, \tau_{2}\right)-\frac{1}{2}\left(r_{i j}\left(\tau_{1}\right)-r_{i j}\left(\tau_{2}\right)\right)^{2} \Delta_{i j}\left(\sigma_{1}, \tau_{1}, \tau_{2}\right) \\
& \quad+\frac{1}{2}\left(\partial_{\tau_{1}}^{2}+\partial_{\tau_{2}}^{2}\right) \Delta_{i j}\left(\sigma_{1}, \tau_{1}, \tau_{2}\right)
\end{aligned}
$$

Let us now divide $\tilde{\Gamma}_{(2)}$ into two parts $\tilde{\Gamma}_{V}$ and $\tilde{\Gamma}_{Y}$ which are expected to correspond to the two contributions of the effective Lagrangian $L_{V}$ and $L_{Y}$ of the previous section, 
respectively.

$$
\begin{gathered}
\tilde{\Gamma}_{(2)}=\tilde{\Gamma}_{V}+\tilde{\Gamma}_{Y} \\
-\tilde{\Gamma}_{V} \\
=\frac{g^{2}}{\kappa} \int d \tau_{1} d \tau_{2} d \sigma_{1} d \sigma_{2} d \sigma_{3} \sum_{i, j, k}\left[-\partial_{\sigma_{1}}\left\{\left(P_{q}+P_{3}\right) \Delta_{i j}\left(\sigma_{1}, \tau_{1}, \tau_{2}\right) \Delta_{j k}\left(\sigma_{2}, \tau_{1}, \tau_{2}\right) \Delta_{k i}\left(\sigma_{3}, \tau_{1}, \tau_{2}\right)\right\}\right],
\end{gathered}
$$

and

$$
\begin{aligned}
& -\tilde{\Gamma}_{Y}=\frac{g^{2}}{\kappa} \int d \tau_{1} d \tau_{2} d \sigma_{1} d \sigma_{2} d \sigma_{3} \sum_{i, j, k}\left[P_{1}\left\{\partial_{\tau_{1}} \partial_{\tau_{2}} \Delta_{i j}\left(\sigma_{1}, \tau_{1}, \tau_{2}\right)\right\} \Delta_{j k}\left(\sigma_{2}, \tau_{1}, \tau_{2}\right) \Delta_{k i}\left(\sigma_{3}, \tau_{1}, \tau_{2}\right)\right. \\
& +P_{2}\left\{\partial_{\tau_{1}} \Delta_{i j}\left(\sigma_{1}, \tau_{1}, \tau_{2}\right)\right\} \Delta_{j k}\left(\sigma_{2}, \tau_{1}, \tau_{2}\right) \Delta_{k i}\left(\sigma_{3}, \tau_{1}, \tau_{2}\right) \\
& -\frac{1}{2} P_{3}\left(r_{i j}\left(\tau_{1}\right)-r_{i j}\left(\tau_{2}\right)\right)^{2} \Delta_{i j}\left(\sigma_{1}, \tau_{1}, \tau_{2}\right) \Delta_{j k}\left(\sigma_{2}, \tau_{1}, \tau_{2}\right) \Delta_{k i}\left(\sigma_{3}, \tau_{1}, \tau_{2}\right) \\
& +\frac{1}{2} P_{3}\left\{\left(\partial_{\tau_{1}}^{2}+\partial_{\tau_{2}}^{2}\right) \Delta_{i j}\left(\sigma_{1}, \tau_{1}, \tau_{2}\right)\right\} \Delta_{j k}\left(\sigma_{2}, \tau_{1}, \tau_{2}\right) \Delta_{k i}\left(\sigma_{3}, \tau_{1}, \tau_{2}\right) \\
& \left.+\left(P_{4}+\partial_{\sigma_{1}} P_{3}\right) \Delta_{i j}\left(\sigma_{1}, \tau_{1}, \tau_{2}\right) \Delta_{j k}\left(\sigma_{2}, \tau_{1}, \tau_{2}\right) \Delta_{k i}\left(\sigma_{3}, \tau_{1}, \tau_{2}\right)\right] .
\end{aligned}
$$

Since the whole dependence on $\left\{x^{2}\right\}$ in $\tilde{\Gamma}_{V}$ and $\tilde{\Gamma}_{Y}$ is now contained only in the proper-time propagators, we can expect that the above cancellation could occur before the proper-time integrations, that is, in the prefactors in front of proper-time propagators. This is indeed the case.

\subsubsection{The calculation of $\tilde{\Gamma}_{V}$}

Let us begin with $\tilde{\Gamma}_{V}$. It is easily calculated as follows:

$$
\begin{aligned}
\tilde{\Gamma}_{V}=- & \frac{g^{2}}{\kappa} \sum_{i, j, k} \int_{-\infty}^{\infty} d \tau \int_{0}^{\infty} d \sigma_{2} \int_{0}^{\infty} d \sigma_{3} 128 \sinh ^{3} \frac{\sigma_{2} \tilde{v}_{j k}}{2} \sinh ^{3} \frac{\sigma_{3} \tilde{v}_{k i}}{2} \\
& \times\left(\frac{2 \tilde{v}_{j k} \cdot \tilde{v}_{k i}}{\tilde{v}_{j k} \tilde{v}_{k i}} \cosh \frac{\sigma_{2} \tilde{v}_{j k}}{2} \cosh \frac{\sigma_{3} \tilde{v}_{k i}}{2}-\sinh \frac{\sigma_{2} \tilde{v}_{j k}}{2} \sinh \frac{\sigma_{3} \tilde{v}_{k i}}{2}\right) \\
& \times \Delta_{j k}\left(\sigma_{2}, \tau, \tau\right) \Delta_{k i}\left(\sigma_{3}, \tau, \tau\right) \\
= & -\frac{g^{2}}{\kappa} \sum_{i, j, k} \int_{0}^{\infty} d \sigma_{2} \int_{0}^{\infty} d \sigma_{3} 128 \sinh ^{3} \frac{\sigma_{2} \tilde{v}_{j k}}{2} \sinh ^{3} \frac{\sigma_{3} \tilde{v}_{k i}}{2}
\end{aligned}
$$




$$
\begin{aligned}
& \times\left(\frac{2 \tilde{v}_{j k} \cdot \tilde{v}_{k i}}{\tilde{v}_{j k} \tilde{v}_{k i}} \cosh \frac{\sigma_{2} \tilde{v}_{j k}}{2} \cosh \frac{\sigma_{3} \tilde{v}_{k i}}{2}-\sinh \frac{\sigma_{2} \tilde{v}_{j k}}{2} \sinh \frac{\sigma_{3} \tilde{v}_{k i}}{2}\right) \\
& \times \sqrt{\frac{\tilde{v}_{j k}}{2 \pi \sinh \left(2 \sigma_{2} \tilde{v}_{j k}\right)}} \sqrt{\frac{\tilde{v}_{k i}}{2 \pi \sinh \left(2 \sigma_{3} \tilde{v}_{k i}\right)}} \sqrt{\frac{\pi}{\tilde{v}_{j k} \tanh \left(\sigma_{2} \tilde{v}_{j k}\right)+\tilde{v}_{k i} \tanh \left(\sigma_{3} \tilde{v}_{k i}\right)}} \\
& \times \exp \left[-\sigma_{2}\left(x_{j k}^{2}-\frac{\left(x_{j k} \cdot \tilde{v}_{j k}\right)^{2}}{\tilde{v}_{j k}^{2}}\right)-\sigma_{3}\left(x_{k i}^{2}-\frac{\left(x_{k i} \cdot \tilde{v}_{k i}\right)^{2}}{\tilde{v}_{k i}^{2}}\right)\right. \\
& \left.-\frac{\tilde{v}_{j k} \tilde{v}_{k i} \tanh \left(\sigma_{2} \tilde{v}_{j k}\right) \tanh \left(\sigma_{3} \tilde{v}_{k i}\right)}{\tilde{v}_{j k} \tanh \left(\sigma_{2} \tilde{v}_{j k}\right)+\tilde{v}_{k i} \tanh \left(\sigma_{3} \tilde{v}_{k i}\right)}\left(\frac{x_{j k} \cdot \tilde{v}_{j k}}{\tilde{v}_{j k}^{2}}-\frac{x_{k i} \cdot \tilde{v}_{k i}}{\tilde{v}_{k i}^{2}}\right)^{2}\right]
\end{aligned}
$$

There indeed happened nontrivial cancellations in the prefactor in front of the proper-time propagators and it is of order $O\left(\tilde{v}^{6}\right)$. The expression for $\tilde{\Gamma}_{V}$ (3.44) is exact and completely general just as that for $\tilde{\Gamma}_{(1)}(3.29)$. We want to emphasize that this general expression already shows the characteristic kinematical structure which is consistent with the result $L_{V}$ of supergravity, and that there arises no infrared divergence in the limit of coincident D-particles.

The leading contribution of $\tilde{\Gamma}_{V}$ takes the form

$$
\begin{aligned}
\tilde{\Gamma}_{V \text { leading }}= & -\frac{g^{2}}{\kappa} \sum_{i, j, k} \int_{-\infty}^{\infty} d \tau \int_{0}^{\infty} d \sigma_{2} \int_{0}^{\infty} d \sigma_{3} 4 \sigma_{2}^{3} \sigma_{3}^{3} \tilde{v}_{j k}^{2} \tilde{v}_{k i}^{2}\left(\tilde{v}_{j k} \cdot \tilde{v}_{k i}\right) \\
& \times \frac{1}{4 \pi \sqrt{\sigma_{2} \sigma_{3}}} \exp \left[-\sigma_{2}\left(\tilde{v}_{j k} \tau+x_{j k}\right)^{2}-\sigma_{3}\left(\tilde{v}_{k i} \tau+x_{k i}\right)^{2}\right] \\
= & -\frac{g^{2}}{\kappa} \sum_{i, j, k} \frac{225}{64} \int_{-\infty}^{\infty} d \tau \frac{\tilde{v}_{j k}^{2} \tilde{v}_{k i}^{2}\left(\tilde{v}_{j k} \cdot \tilde{v}_{k i}\right)}{\left[\left(\tilde{v}_{j k} \tau+x_{j k}\right)^{2}\right]^{7 / 2}\left[\left(\tilde{v}_{k i} \tau+x_{k i}\right)^{2}\right]^{7 / 2}}
\end{aligned}
$$

where we used the proper-time representation (3.31).

\subsubsection{The calculation of $\tilde{\Gamma}_{Y}$}

We now proceed to $\tilde{\Gamma}_{Y}$. We can eliminate the time derivatives acting on $\Delta_{i j}\left(\sigma_{1}, \tau_{1}, \tau_{2}\right)$ using the formulas given in Appendix C. The result is then

$$
\begin{array}{r}
-\tilde{\Gamma}_{Y}=\frac{g^{2}}{\kappa} \int d \tau_{1} d \tau_{2} d \sigma_{1} d \sigma_{2} d \sigma_{3} \sum_{i, j, k} P_{Y}\left(\{s\},\{\tilde{v}\},\{x \cdot \tilde{v}\}, \tau_{1}, \tau_{2}\right) \\
\times \Delta_{i j}\left(\sigma_{1}, \tau_{1}, \tau_{2}\right) \Delta_{j k}\left(\sigma_{2}, \tau_{1}, \tau_{2}\right) \Delta_{k i}\left(\sigma_{3}, \tau_{1}, \tau_{2}\right)
\end{array}
$$

where

$$
P_{Y}\left(\{s\},\{\tilde{v}\},\{x \cdot \tilde{v}\}, \tau_{1}, \tau_{2}\right)
$$




$$
\begin{aligned}
=P_{1}[- & \frac{\tilde{v}_{i j}}{2} \frac{V_{i j}\left(\sigma_{1}\right)}{C_{i j}\left(\sigma_{1}\right)}+\frac{\tilde{v}_{i j}}{2} \frac{C_{i j}\left(\sigma_{1}\right)}{V_{i j}\left(\sigma_{1}\right)}+\left(\frac{V_{i j}\left(\sigma_{1}\right)}{C_{i j}\left(\sigma_{1}\right)} \cdot \frac{r_{i j}\left(\tau_{1}\right)+r_{i j}\left(\tau_{2}\right)}{2}\right)^{2} \\
& \left.-\left(\frac{C_{i j}\left(\sigma_{1}\right)}{V_{i j}\left(\sigma_{1}\right)} \frac{r_{i j}\left(\tau_{1}\right)-r_{i j}\left(\tau_{2}\right)}{2}\right)^{2}\right] \\
+P_{2} & {\left[-\frac{V_{i j}\left(\sigma_{1}\right)}{C_{i j}\left(\sigma_{1}\right)} \cdot \frac{r_{i j}\left(\tau_{1}\right)+r_{i j}\left(\tau_{2}\right)}{2}-\frac{C_{i j}\left(\sigma_{1}\right)}{V_{i j}\left(\sigma_{1}\right)} \frac{\left|r_{i j}\left(\tau_{1}\right)-r_{i j}\left(\tau_{2}\right)\right|}{2}\right] } \\
+P_{3} & {\left[-2\left(\frac{r_{i j}\left(\tau_{1}\right)-r_{i j}\left(\tau_{2}\right)}{2}\right)^{2}-\frac{\tilde{v}_{i j}}{2} \frac{V_{i j}\left(\sigma_{1}\right)}{C_{i j}\left(\sigma_{1}\right)}-\frac{\tilde{v}_{i j}}{2} \frac{C_{i j}\left(\sigma_{1}\right)}{V_{i j}\left(\sigma_{1}\right)}\right.} \\
+ & \left.\left(\frac{V_{i j}\left(\sigma_{1}\right)}{C_{i j}\left(\sigma_{1}\right)} \cdot \frac{r_{i j}\left(\tau_{1}\right)+r_{i j}\left(\tau_{2}\right)}{2}\right)^{2}+\left(\frac{C_{i j}\left(\sigma_{1}\right)}{V_{i j}\left(\sigma_{1}\right)} \frac{r_{i j}\left(\tau_{1}\right)-r_{i j}\left(\tau_{2}\right)}{2}\right)^{2}\right] \\
+ & P_{4}+\partial_{\sigma_{1}} P_{3} .
\end{aligned}
$$

We have made no approximations for $\tilde{\Gamma}_{Y}$ so far and we can carry out the integrations with respect to $\tau_{1}$ and $\tau_{2}$ as well. Thus, we can in principle evaluate the leading contribution with respect to $\{\tilde{v}\}$ simply by expanding it to Taylor series. However, the expression is too complicated to do that in a completely general way. So we content ourselves to restrict to the cases where $\{x \cdot \tilde{v}\}$ vanish. Since the relative velocities $\tilde{v}_{i j}$ are independent of the relative coordinates $x_{i j}$ at initial time $\tau=0$, we can arrange, for arbitrary given configurations of relative velocities, the trajectories of D-particles by making parallel transport of the positions of each D-particle at time $\tau=0$ appropriately such that the conditions are satisfied for three D-particles which participate in the 3-body interaction in 9 transverse dimensions. Then the integrations with respect to $\tau_{1}$ and $\tau_{2}$ are considerably simplified. Using the formulas in Appendix $\mathrm{C}, \tilde{\Gamma}_{Y}$ can be rearranged into the form

$$
\begin{array}{r}
-\tilde{\Gamma}_{Y}=\frac{g^{2}}{\kappa} \int d \tau_{1} d \tau_{2} d \sigma_{1} d \sigma_{2} d \sigma_{3} \sum_{i, j, k} \tilde{P}_{Y}\left(\sigma_{1}, \sigma_{2}, \sigma_{3}, \tilde{v}_{i j}, \tilde{v}_{j k}, \tilde{v}_{k i}\right) \\
\times \Delta_{i j}\left(\sigma_{1}, \tau_{1}, \tau_{2}\right) \Delta_{j k}\left(\sigma_{2}, \tau_{1}, \tau_{2}\right) \Delta_{k i}\left(\sigma_{3}, \tau_{1}, \tau_{2}\right),
\end{array}
$$

where

$$
\begin{aligned}
\tilde{P}_{Y}\left(\sigma_{1}, \sigma_{2}, \sigma_{3}, \tilde{v}_{i j}, \tilde{v}_{j k}, \tilde{v}_{k i}\right)=P_{1}\left[-\frac{\tilde{v}_{i j}}{2} \frac{V_{i j}\left(\sigma_{1}\right)}{C_{i j}\left(\sigma_{1}\right)}+\frac{\tilde{v}_{i j}}{2} \frac{C_{i j}\left(\sigma_{1}\right)}{V_{i j}\left(\sigma_{1}\right)}\right] \\
+P_{3}\left[-\frac{\tilde{v}_{i j}}{2} \frac{V_{i j}\left(\sigma_{1}\right)}{C_{i j}\left(\sigma_{1}\right)}-\frac{\tilde{v}_{i j}}{2} \frac{C_{i j}\left(\sigma_{1}\right)}{V_{i j}\left(\sigma_{1}\right)}\right]+\partial_{\sigma_{1}} P_{3} \\
+\frac{1}{2}\left(\frac{\tilde{v}_{i j} V_{i j}\left(\sigma_{1}\right)}{C_{i j}\left(\sigma_{1}\right)}+\frac{\tilde{v}_{j k} V_{j k}\left(\sigma_{2}\right)}{C_{j k}\left(\sigma_{2}\right)}+\frac{\tilde{v}_{k i} V_{k i}\left(\sigma_{3}\right)}{C_{k i}\left(\sigma_{3}\right)}\right)^{-1}
\end{aligned}
$$




$$
\begin{aligned}
& {\left[P_{1}\left(\frac{\tilde{v}_{i j} V_{i j}\left(\sigma_{1}\right)}{C_{i j}\left(\sigma_{1}\right)}\right)^{2}-\frac{P_{2}}{\tau_{2}} \frac{\tilde{v}_{i j} V_{i j}\left(\sigma_{1}\right)}{C_{i j}\left(\sigma_{1}\right)}+P_{3}\left(\frac{\tilde{v}_{i j} V_{i j}\left(\sigma_{1}\right)}{C_{i j}\left(\sigma_{1}\right)}\right)^{2}+\frac{P_{4}}{\tau_{1} \tau_{2}}\right] } \\
&+\frac{1}{2}\left(\frac{\tilde{v}_{i j} C_{i j}\left(\sigma_{1}\right)}{V_{i j}\left(\sigma_{1}\right)}+\frac{\tilde{v}_{j k} C_{j k}\left(\sigma_{2}\right)}{V_{j k}\left(\sigma_{2}\right)}+\frac{\tilde{v}_{k i} C_{k i}\left(\sigma_{3}\right)}{V_{k i}\left(\sigma_{3}\right)}\right)^{-1} \\
& {\left[-P_{1}\left(\frac{\tilde{v}_{i j} C_{i j}\left(\sigma_{1}\right)}{V_{i j}\left(\sigma_{1}\right)}\right)^{2}+\frac{P_{2}}{\tau_{2}} \frac{\tilde{v}_{i j} C_{i j}\left(\sigma_{1}\right)}{V_{i j}\left(\sigma_{1}\right)}-2 \tilde{v}_{i j}^{2} P_{3}+P_{3}\left(\frac{\tilde{v}_{i j} C_{i j}\left(\sigma_{1}\right)}{V_{i j}\left(\sigma_{1}\right)}\right)^{2}-\frac{P_{4}}{\tau_{1} \tau_{2}}\right] . }
\end{aligned}
$$

We expand $\tilde{P}_{Y}$ with respect to $\tilde{v}_{i j}, \tilde{v}_{j k}$ and $\tilde{v}_{k i}$ after symmetrization. The result is $\mathrm{\theta}$

$$
\begin{aligned}
& \tilde{P}_{Y}\left(\sigma_{1}, \sigma_{2}, \sigma_{3}, \tilde{v}_{i j}, \tilde{v}_{j k}, \tilde{v}_{k i}\right)_{s y m} \\
& =\frac{1}{6}\left[\tilde{P}_{Y}\left(\sigma_{1}, \sigma_{2}, \sigma_{3}, \tilde{v}_{i j}, \tilde{v}_{j k}, \tilde{v}_{k i}\right)+\tilde{P}_{Y}\left(\sigma_{1}, \sigma_{3}, \sigma_{2}, \tilde{v}_{i j}, \tilde{v}_{k i}, \tilde{v}_{j k}\right)+\tilde{P}_{Y}\left(\sigma_{2}, \sigma_{1}, \sigma_{3}, \tilde{v}_{j k}, \tilde{v}_{i j}, \tilde{v}_{k i}\right)\right. \\
& \left.\quad+\tilde{P}_{Y}\left(\sigma_{2}, \sigma_{3}, \sigma_{1}, \tilde{v}_{j k}, \tilde{v}_{k i}, \tilde{v}_{i j}\right)+\tilde{P}_{Y}\left(\sigma_{3}, \sigma_{1}, \sigma_{2}, \tilde{v}_{k i}, \tilde{v}_{i j}, \tilde{v}_{j k}\right)+\tilde{P}_{Y}\left(\sigma_{3}, \sigma_{2}, \sigma_{1}, \tilde{v}_{k i}, \tilde{v}_{j k}, \tilde{v}_{i j}\right)\right] \\
& =-\frac{1}{6}\left(\tilde{v}_{i j}+\tilde{v}_{j k}+\tilde{v}_{k i}\right)\left(-\tilde{v}_{i j}+\tilde{v}_{j k}+\tilde{v}_{k i}\right)\left(\tilde{v}_{i j}-\tilde{v}_{j k}+\tilde{v}_{k i}\right)\left(\tilde{v}_{i j}+\tilde{v}_{j k}-\tilde{v}_{k i}\right) \\
& \quad \times\left(\sigma_{1} \sigma_{2}+\sigma_{2} \sigma_{3}+\sigma_{3} \sigma_{1}\right)^{2}\left(\sigma_{1} \tilde{v}_{i j}^{2}+\sigma_{2} \tilde{v}_{j k}^{2}+\sigma_{3} \tilde{v}_{k i}^{2}\right)+O\left(\tilde{v}^{8}\right) .
\end{aligned}
$$

We again encountered miraculous cancellations here just as in $\tilde{\Gamma}_{V}$. Then, after integrating over the times $\tau_{1}$ and $\tau_{2}$, the leading contribution to $\tilde{\Gamma}_{Y}$ is given by

$$
\begin{aligned}
\tilde{\Gamma}_{Y \text { leading }}=\frac{g^{2}}{\kappa} \sum_{i, j, k} & \int_{0}^{\infty} d \sigma_{1} \int_{0}^{\infty} d \sigma_{2} \int_{0}^{\infty} d \sigma_{3} \frac{1}{24 \sqrt{\pi}}\left(\tilde{v}_{i j}+\tilde{v}_{j k}+\tilde{v}_{k i}\right) \\
& \times\left(-\tilde{v}_{i j}+\tilde{v}_{j k}+\tilde{v}_{k i}\right)\left(\tilde{v}_{i j}-\tilde{v}_{j k}+\tilde{v}_{k i}\right)\left(\tilde{v}_{i j}+\tilde{v}_{j k}-\tilde{v}_{k i}\right) \\
& \times\left(\sigma_{1} \sigma_{2}+\sigma_{2} \sigma_{3}+\sigma_{3} \sigma_{1}\right)^{3 / 2} \sqrt{\sigma_{1} \tilde{v}_{i j}^{2}+\sigma_{2} \tilde{v}_{j k}^{2}+\sigma_{3} \tilde{v}_{k i}^{2}} \\
& \times \exp \left(-\sigma_{1} x_{i j}^{2}-\sigma_{2} x_{j k}^{2}-\sigma_{3} x_{k i}^{2}\right) .
\end{aligned}
$$

This formula vanishes whenever any one of the relative velocities vanishes, since the product

$$
\frac{1}{16}\left(\tilde{v}_{i j}+\tilde{v}_{j k}+\tilde{v}_{k i}\right)\left(-\tilde{v}_{i j}+\tilde{v}_{j k}+\tilde{v}_{k i}\right)\left(\tilde{v}_{i j}-\tilde{v}_{j k}+\tilde{v}_{k i}\right)\left(\tilde{v}_{i j}+\tilde{v}_{j k}-\tilde{v}_{k i}\right)
$$

is just the square of the area, known as Heron's formula, of the triangle formed by three relative velocity vectors $\tilde{v}_{i j}, \tilde{v}_{j k}, \tilde{v}_{k i}$. This is a nontrivial check of our result in conformity with the BPS property of D-particles and consistent with the structure of the 3-body Lagrangian of supergravity. 4.

${ }^{\ddagger}$ We performed this computation with the help of the symbolic computation program Maple V Release 


\section{Comparison between supergravity and Matrix theory}

We are now ready to compare the results of Matrix theory with the prediction of supergravity. To compare the effective action of the Matrix-theory calculations with the result from supergravity, we have first to take into account the changes of conventions in both cases, most of which are already explained in the beginning of section 3 (see below (3.7)). Let us summarize them again here.

First, while we computed the effective action with completely general diagonal backgrounds, we are actually interested in backgrounds consisting of several blocks proportional to unit matrices of the order $N_{a}\left(a=1,2, \ldots, N=\sum_{a} N_{a}\right)$. The summations over $\mathrm{U}(N)$ indices automatically take care of this effect and just reduce to the summation over the block elements. Secondly, since the supergravity calculations are carried out in Minkowski space-time, we have to transform the Euclidean effective action to Minkowski signature. Thirdly, we have to rescale the coordinates and velocities:

$$
\begin{aligned}
& \sum_{i} \rightarrow \sum_{a} N_{a}, \quad \tilde{\Gamma} \rightarrow-i \Gamma, \quad \tau \rightarrow i t, \quad \kappa \rightarrow \frac{1}{R}, \quad g \rightarrow M^{3} R, \\
& x \rightarrow g x=M^{3} R x, \quad \tilde{v} \rightarrow g(-i v)=M^{3} R(-i v), \quad \sigma \rightarrow \frac{\sigma}{g^{2}}=\frac{\sigma}{M^{6} R^{2}} .
\end{aligned}
$$

With these replacements, the Matrix-theory results read

$$
\begin{aligned}
& \Gamma_{(0)}= \int_{-\infty}^{\infty} d t \sum_{a} \frac{1}{2} \frac{N_{a}}{R} v_{a}^{2} \\
& \Gamma_{(1) \text { leading }}= \int_{-\infty}^{\infty} d t \sum_{a<b} \frac{15}{16} \frac{N_{a} N_{b}}{R^{3} M^{9}} \frac{v_{a b}^{4}}{\left[\left(v_{a b} t+x_{a b}\right)^{2}\right]^{7 / 2}}, \\
& \Gamma_{V \text { leading }}=-\int_{-\infty}^{\infty} d t \sum_{a, b, c} \frac{225}{64} \frac{N_{a} N_{b} N_{c}}{R^{5} M^{18}} \frac{v_{b c}^{2} v_{c a}^{2}\left(v_{b c} \cdot v_{c a}\right)}{\left[\left(v_{b c} t+x_{b c}\right)^{2}\right]^{7 / 2}\left[\left(v_{c a} t+x_{c a}\right)^{2}\right]^{7 / 2}}, \\
& \Gamma_{Y \text { leading }=} \int_{0}^{\infty} d \sigma_{1} \int_{0}^{\infty} d \sigma_{2} \int_{0}^{\infty} d \sigma_{3} \sum_{a, b, c} \frac{1}{24 \sqrt{\pi}} \frac{N_{a} N_{b} N_{c}}{R^{5} M^{18}}\left(v_{a b}+v_{b c}+v_{c a}\right) \\
& \times\left(-v_{a b}+v_{b c}+v_{c a}\right)\left(v_{a b}-v_{b c}+v_{c a}\right)\left(v_{a b}+v_{b c}-v_{c a}\right) \\
& \times\left(\sigma_{1} \sigma_{2}+\sigma_{2} \sigma_{3}+\sigma_{3} \sigma_{1}\right)^{3 / 2} \sqrt{\sigma_{1} v_{a b}^{2}+\sigma_{2} v_{b c}^{2}+\sigma_{3} v_{c a}^{2}} \\
& \times \exp \left(-\sigma_{1} x_{a b}^{2}-\sigma_{2} x_{b c}^{2}-\sigma_{3} x_{c a}^{2}\right) .
\end{aligned}
$$

Recall that the results $\Gamma_{(0)}, \Gamma_{(1)}$ leading and $\Gamma_{V}$ leading are for completely general situations, while $\Gamma_{Y}$ leading is for the case where $\{x \cdot v\}$ vanish. Note, however, as already emphasized 
before, that the latter condition can be satisfied for arbitrary configurations of relative velocities of three D-particles, provided that only the relative coordinates at initial time are chosen appropriately.

We turn to computations of the phase shift in the eikonal approximation using the effective Lagrangian obtained in supergravity . As for the 2-body Lagrangian $L_{2}$ (2.50) and the $L_{V}$ part (2.53) of the 3-body Lagrangian, it is already evident that they reproduce exactly the same phase shifts as those of Matrix theory (4.3) and (4.4), since the integrands in these expressions are identical with the corresponding effective Lagrangians of supergravity.

For the $L_{Y}$ part, the result in Matrix theory is given in a form where the integration over time has been performed. Thus the relation with supergravity is not obvious. Let us therefore explicitly compute the eikonal phase shift from the $L_{Y}$ part of the 3-body Lagrangian. We compute it with the same restriction $\{x \cdot v\}=0$ as we have made for $\Gamma_{Y}$ in Matrix theory, assuming the straight-line trajectories $x_{a}(\tau)=x_{a}+v_{a} \tau$. Then by a tedious but straightforward calculation, including symmetrization with respect to the block indices $a, b$ and $c$, we can arrange the time integral of the Lagrangian $L_{Y}$ to the following form

$$
\begin{aligned}
& -\int_{-\infty}^{\infty} d \tau \sum_{a, b, c} \frac{(15)^{3} N_{a} N_{b} N_{c}}{96(2 \pi)^{4}}\left[-v_{b c}^{2} v_{c a}^{2}\left(v_{c b} \cdot \nabla_{c}\right)\left(v_{c a} \cdot \nabla_{c}\right)\right. \\
& +\frac{1}{2} v_{c a}^{4}\left(v_{c b} \cdot \nabla_{c}\right)^{2}+\frac{1}{2} v_{b c}^{4}\left(v_{c a} \cdot \nabla_{c}\right)^{2}-\frac{1}{2} v_{b a}^{2} v_{a c}^{2}\left(v_{c b} \cdot \nabla_{c}\right)\left(v_{b c} \cdot \nabla_{b}\right) \\
& \left.+\frac{1}{4} v_{b c}^{4}\left(v_{b a} \cdot \nabla_{b}\right)\left(v_{c a} \cdot \nabla_{c}\right)\right] \Delta(a, b, c) \\
& =\int_{-\infty}^{\infty} d \tau \sum_{a, b, c} \frac{N_{a} N_{b} N_{c}}{24 \pi} \int_{0}^{\infty} d^{3} \sigma\left(\sigma_{1} \sigma_{2}+\sigma_{2} \sigma_{3}+\sigma_{3} \sigma_{1}\right)^{3 / 2} \\
& \quad \times\left(v_{a b}+v_{b c}+v_{c a}\right)\left(-v_{a b}+v_{b c}+v_{c a}\right)\left(v_{a b}-v_{b c}+v_{c a}\right)\left(v_{a b}+v_{b c}-v_{c a}\right) \\
& \quad \times\left(\sigma_{1} v_{a b}^{2}+\sigma_{2} v_{b c}^{2}+\sigma_{3} v_{c a}^{2}\right) \exp \left(-\sigma_{1} x_{a b}^{2}-\sigma_{2} x_{b c}^{2}-\sigma_{3} x_{c a}^{2}\right) .
\end{aligned}
$$

Performing the time integral explicitly gives the following expression for the phase shift:

$$
\begin{aligned}
\int_{0}^{\infty} & d \sigma_{1} \int_{0}^{\infty} d \sigma_{2} \int_{0}^{\infty} d \sigma_{3} \sum_{a, b, c} \frac{1}{24 \sqrt{\pi}} \frac{N_{a} N_{b} N_{c}}{R^{5} M^{18}}\left(v_{a b}+v_{b c}+v_{c a}\right) \\
& \times\left(-v_{a b}+v_{b c}+v_{c a}\right)\left(v_{a b}-v_{b c}+v_{c a}\right)\left(v_{a b}+v_{b c}-v_{c a}\right) \\
& \times\left(\sigma_{1} \sigma_{2}+\sigma_{2} \sigma_{3}+\sigma_{3} \sigma_{1}\right)^{3 / 2} \sqrt{\sigma_{1} v_{a b}^{2}+\sigma_{2} v_{b c}^{2}+\sigma_{3} v_{c a}^{2}}
\end{aligned}
$$




$$
\times \exp \left(-\sigma_{1} x_{a b}^{2}-\sigma_{2} x_{b c}^{2}-\sigma_{3} x_{c a}^{2}\right)
$$

which precisely coincides with $\Gamma_{Y}$ leading from Matrix theory including the sign and numerical coefficient. Since, in this calculation, all the terms in the left hand side in (4.6) contribute in a nontrivial way, we are confident that the agreement will extend to the general case where the technical restriction $\{x \cdot v\}=0$ is not assumed.

It should be emphasized that in both of our supergravity and Matrix theory calculations, the recoil effect is neglected completely. In order to take into account the recoil effect, we have to allow non-local (in time) contributions to the effective action in supergravity and the 1-particle reducible contributions in the phase shift of Matrix theory. We hope to report on the recoil effect in a forthcoming work.

\section{Concluding Remarks}

We have presented a detailed study of the 3-body interaction of D-particles and established its precise equivalence in Matrix theory and 11 dimensional supergravity. Our results provide a strong support for the idea [14] that the Matrix theory is a formulation of M-theory in the discrete light-cone quantization. As emphasized in the Introduction, we also view our results as evidence for Lorentz invariance of Matrix theory. In a sense, what we have done in the present paper may be regarded as a Matrix-theory analogue to the old works [15] 16] in which the non-linear graviton behavior in string theory was first established. In the case of the usual string theory, the formalism is Lorentz invariant from the beginning and hence the emergence of Einstein gravity is ensured by checking the non-linear effect to the first nontrivial order, owing to the general theorem (see, e. g. [17]) showing the uniqueness of the Einstein equation. Because of this, we could be fairly confident in that the string theory describes gravity in a consistent way in the long-distance limit. In the case of Matrix theory, unfortunately, Lorentz invariant formulation still remains as a big mystery. Perhaps one of the most crucial issue in the connection of Matrix theory and supergravity is to establish a more systematic way of making correspondence between supergravity and Matrix theory. Technically, for example, it may be possible to relate both theories order by order at the level of Feynman rules. There should be some correspondence principle which ensures that the long-distance property of Matrix theory 
is reproduced by supergravity.

Finally, in view of the recent discovery [18] of the conformal symmetry associated with the space-time uncertainty relation for D-particles [19], it is interesting to see whether there exists a generalization of the conformal symmetry for our effective action for many D-particle systems. Such a symmetry, if it exists, combined with the supersymmetry is expected to determine the form of the effective action to all orders in the velocity expansion in the classical limit. A related problem is the supersymmetric generalization of our effective action to include the spin degrees of freedom.

\section{Note added}

After the completion of the present work, in the course of preparing the manuscript, two papers [20] 21] discussing the same subject appeared in hep-th archive. However, both of them reported only the considerations of particular subsets of the relevant contributions and hence remain inconclusive as to the agreement between supergravity and Matrix theory. We believe that the present work gives a final resolution on the issue.

\section{Acknowledgements}

We would like to thank M. Ikehara for discussions on two-loop computations in matrix models and, especially, to Y. Kazama for collaborative discussions on the role of supersymmetry in the dynamics of D-particles at an early stage of the present work. A part of the present work was done during one (T. Y.) of the authors was staying at ITP, Santa Barbara. T.Y. would like to thank ITP for its hospitality. The research at ITP was supported in part by the National Science Foundation under Grant No. PHY94-07194. The work of T.Y. is supported in part by Grant-in-Aid for Scientific Research (No. 09640337) and Grant-in-Aid for International Scientific Research (Joint Research, No. 10044061) from the Ministry of Education, Science and Culture. The work of Y. O. is supported in part by the Japan Society for the Promotion of Science under the Predoctoral Research Program (No. 08-4158). 


\section{Appendix}

\section{A Explicit forms of $P_{q}, P_{1}, P_{2}, P_{3}$ and $P_{4}$}

We here present the explicit forms of $P_{q}, P_{1}, P_{2}, P_{3}$ and $P_{4}$.

$$
\begin{aligned}
& P_{q}=-45-18 V_{j k}\left(\sigma_{2}\right)^{2}-18 V_{k i}\left(\sigma_{3}\right)^{2}+12 C_{j k}\left(\sigma_{2}\right) C_{k i}\left(\sigma_{3}\right)\left(V_{j k}\left(\sigma_{2}\right) \cdot V_{k i}\left(\sigma_{3}\right)\right) \\
& -6 V_{j k}\left(\sigma_{2}\right)^{2} V_{k i}\left(\sigma_{3}\right)^{2}+2\left(V_{j k}\left(\sigma_{2}\right) \cdot V_{k i}\left(\sigma_{3}\right)\right)^{2} \text {, } \\
& P_{1}=-\left(1+2 V_{i j}\left(\sigma_{1}\right)^{2}\right)\left\{4+V_{j k}\left(\sigma_{2}\right)^{2}+V_{k i}\left(\sigma_{3}\right)^{2}+2\left(V_{j k}\left(\sigma_{2}\right) \cdot V_{k i}\left(\sigma_{3}\right)\right)^{2}\right\} \\
& +\left(1+2 V_{j k}\left(\sigma_{2}\right)^{2}\right)\left\{\frac{17}{2}+2 V_{i j}\left(\sigma_{1}\right)^{2}+2 V_{k i}\left(\sigma_{3}\right)^{2}+4\left(V_{i j}\left(\sigma_{1}\right) \cdot V_{k i}\left(\sigma_{3}\right)\right)^{2}\right\} \\
& +\left(1+2 V_{k i}\left(\sigma_{3}\right)^{2}\right)\left\{\frac{17}{2}+2 V_{i j}\left(\sigma_{1}\right)^{2}+2 V_{j k}\left(\sigma_{2}\right)^{2}+4\left(V_{i j}\left(\sigma_{1}\right) \cdot V_{j k}\left(\sigma_{2}\right)\right)^{2}\right\} \\
& -10 C_{j k}\left(\sigma_{2}\right) C_{k i}\left(\sigma_{3}\right)\left\{V_{j k}\left(\sigma_{2}\right) \cdot V_{k i}\left(\sigma_{3}\right)+2\left(V_{i j}\left(\sigma_{1}\right) \cdot V_{j k}\left(\sigma_{2}\right)\right)\left(V_{i j}\left(\sigma_{1}\right) \cdot V_{k i}\left(\sigma_{3}\right)\right)\right\} \\
& +2 C_{i j}\left(\sigma_{1}\right) C_{j k}\left(\sigma_{2}\right)\left\{V_{i j}\left(\sigma_{1}\right) \cdot V_{j k}\left(\sigma_{2}\right)+2\left(V_{i j}\left(\sigma_{1}\right) \cdot V_{k i}\left(\sigma_{3}\right)\right)\left(V_{j k}\left(\sigma_{2}\right) \cdot V_{k i}\left(\sigma_{3}\right)\right)\right\} \\
& +2 C_{i j}\left(\sigma_{1}\right) C_{k i}\left(\sigma_{3}\right)\left\{V_{i j}\left(\sigma_{1}\right) \cdot V_{k i}\left(\sigma_{3}\right)+2\left(V_{i j}\left(\sigma_{1}\right) \cdot V_{j k}\left(\sigma_{2}\right)\right)\left(V_{j k}\left(\sigma_{2}\right) \cdot V_{k i}\left(\sigma_{3}\right)\right)\right\} \\
& +16\left\{-2 C_{j k}\left(\sigma_{2}\right) C_{k i}\left(\sigma_{3}\right)-2 V_{j k}\left(\sigma_{2}\right) \cdot V_{k i}\left(\sigma_{3}\right)-V_{i j}\left(\sigma_{1}\right)^{2}\left(V_{j k}\left(\sigma_{2}\right) \cdot V_{k i}\left(\sigma_{3}\right)\right)\right. \\
& +\left(V_{i j}\left(\sigma_{1}\right) \cdot V_{j k}\left(\sigma_{2}\right)\right)\left(V_{i j}\left(\sigma_{1}\right) \cdot V_{k i}\left(\sigma_{3}\right)\right) \\
& +2 C_{i j}\left(\sigma_{1}\right) C_{k i}\left(\sigma_{3}\right)+2 V_{i j}\left(\sigma_{1}\right) \cdot V_{k i}\left(\sigma_{3}\right)+V_{j k}\left(\sigma_{2}\right)^{2}\left(V_{i j}\left(\sigma_{1}\right) \cdot V_{k i}\left(\sigma_{3}\right)\right) \\
& -\left(V_{i j}\left(\sigma_{1}\right) \cdot V_{j k}\left(\sigma_{2}\right)\right)\left(V_{j k}\left(\sigma_{2}\right) \cdot V_{k i}\left(\sigma_{3}\right)\right) \\
& +2 C_{i j}\left(\sigma_{1}\right) C_{j k}\left(\sigma_{2}\right)+2 V_{i j}\left(\sigma_{1}\right) \cdot V_{j k}\left(\sigma_{2}\right)+V_{k i}\left(\sigma_{3}\right)^{2}\left(V_{i j}\left(\sigma_{1}\right) \cdot V_{j k}\left(\sigma_{2}\right)\right) \\
& \left.-\left(V_{i j}\left(\sigma_{1}\right) \cdot V_{k i}\left(\sigma_{3}\right)\right)\left(V_{j k}\left(\sigma_{2}\right) \cdot V_{k i}\left(\sigma_{3}\right)\right)\right\} \text {, } \\
& P_{2}=-4 C_{i j}\left(\sigma_{1}\right)\left(V_{i j}\left(\sigma_{1}\right) \cdot r_{i j}\left(\tau_{2}\right)\right) \\
& \times\left\{4+V_{j k}\left(\sigma_{2}\right)^{2}+V_{k i}\left(\sigma_{3}\right)^{2}+2\left(V_{j k}\left(\sigma_{2}\right) \cdot V_{k i}\left(\sigma_{3}\right)\right)^{2}\right. \\
& \left.-2 C_{j k}\left(\sigma_{2}\right) C_{k i}\left(\sigma_{3}\right)\left(V_{j k}\left(\sigma_{2}\right) \cdot V_{k i}\left(\sigma_{3}\right)\right)\right\} \\
& +4 C_{j k}\left(\sigma_{2}\right)\left(V_{j k}\left(\sigma_{2}\right) \cdot r_{i j}\left(\tau_{2}\right)\right) \\
& \times\left\{\frac{17}{2}+2 V_{i j}\left(\sigma_{1}\right)^{2}+2 V_{k i}\left(\sigma_{3}\right)^{2}+4\left(V_{i j}\left(\sigma_{1}\right) \cdot V_{k i}\left(\sigma_{3}\right)\right)^{2}\right. \\
& \left.-4 C_{i j}\left(\sigma_{1}\right) C_{k i}\left(\sigma_{3}\right)\left(V_{i j}\left(\sigma_{1}\right) \cdot V_{k i}\left(\sigma_{3}\right)\right)\right\} \\
& +4 C_{k i}\left(\sigma_{3}\right)\left(V_{k i}\left(\sigma_{3}\right) \cdot r_{i j}\left(\tau_{2}\right)\right)
\end{aligned}
$$




$$
\begin{aligned}
& \times\left\{\frac{17}{2}+2 V_{i j}\left(\sigma_{1}\right)^{2}+2 V_{j k}\left(\sigma_{2}\right)^{2}+4\left(V_{i j}\left(\sigma_{1}\right) \cdot V_{j k}\left(\sigma_{2}\right)\right)^{2}\right. \\
& \left.-4 C_{i j}\left(\sigma_{1}\right) C_{j k}\left(\sigma_{2}\right)\left(V_{i j}\left(\sigma_{1}\right) \cdot V_{j k}\left(\sigma_{2}\right)\right)\right\} \\
& +4 C_{j k}\left(\sigma_{2}\right) V_{j k}\left(\sigma_{2}\right) \cdot\left(r_{j k}\left(\tau_{2}\right)-r_{i j}\left(\tau_{2}\right)\right)+4 C_{k i}\left(\sigma_{3}\right) V_{k i}\left(\sigma_{3}\right) \cdot\left(r_{j k}\left(\tau_{2}\right)-r_{k i}\left(\tau_{2}\right)\right) \\
& +4\left\{C_{i j}\left(\sigma_{1}\right)-2 C_{j k}\left(\sigma_{2}\right)\left(C_{i j}\left(\sigma_{1}\right) C_{j k}\left(\sigma_{2}\right)-V_{i j}\left(\sigma_{1}\right) \cdot V_{j k}\left(\sigma_{2}\right)\right)\right\} V_{i j}\left(\sigma_{1}\right) \cdot\left(r_{j k}\left(\tau_{2}\right)-r_{i j}\left(\tau_{2}\right)\right) \\
& +4\left\{C_{j k}\left(\sigma_{2}\right)-2 C_{k i}\left(\sigma_{3}\right)\left(C_{j k}\left(\sigma_{2}\right) C_{k i}\left(\sigma_{3}\right)-V_{j k}\left(\sigma_{2}\right) \cdot V_{k i}\left(\sigma_{3}\right)\right)\right\} V_{j k}\left(\sigma_{2}\right) \cdot\left(r_{j k}\left(\tau_{2}\right)-r_{k i}\left(\tau_{2}\right)\right) \\
& +8\left\{C_{j k}\left(\sigma_{2}\right)\left(V_{j k}\left(\sigma_{2}\right) \cdot V_{k i}\left(\sigma_{3}\right)\right)+C_{i j}\left(\sigma_{1}\right)\left(V_{i j}\left(\sigma_{1}\right) \cdot V_{k i}\left(\sigma_{3}\right)\right)\right. \\
& \left.-2 C_{j k}\left(\sigma_{2}\right)\left(V_{i j}\left(\sigma_{1}\right) \cdot V_{k i}\left(\sigma_{3}\right)\right)\left(C_{i j}\left(\sigma_{1}\right) C_{j k}\left(\sigma_{2}\right)-V_{i j}\left(\sigma_{1}\right) \cdot V_{j k}\left(\sigma_{2}\right)\right)\right\} \\
& \times V_{k i}\left(\sigma_{3}\right) \cdot\left(r_{j k}\left(\tau_{2}\right)-r_{i j}\left(\tau_{2}\right)\right) \\
& +8\left\{C_{k i}\left(\sigma_{3}\right)\left(V_{i j}\left(\sigma_{1}\right) \cdot V_{k i}\left(\sigma_{3}\right)\right)+C_{j k}\left(\sigma_{2}\right)\left(V_{i j}\left(\sigma_{1}\right) \cdot V_{j k}\left(\sigma_{2}\right)\right)\right. \\
& \left.-2 C_{k i}\left(\sigma_{3}\right)\left(V_{i j}\left(\sigma_{1}\right) \cdot V_{j k}\left(\sigma_{2}\right)\right)\left(C_{j k}\left(\sigma_{2}\right) C_{k i}\left(\sigma_{3}\right)-V_{j k}\left(\sigma_{2}\right) \cdot V_{k i}\left(\sigma_{3}\right)\right)\right\} \\
& \times V_{i j}\left(\sigma_{1}\right) \cdot\left(r_{j k}\left(\tau_{2}\right)-r_{k i}\left(\tau_{2}\right)\right) \\
& +64\left\{-2 C_{i j}\left(\sigma_{1}\right)\left(V_{j k}\left(\sigma_{2}\right) \cdot r_{j k}\left(\tau_{2}\right)\right)-C_{j k}\left(\sigma_{2}\right)\left(V_{i j}\left(\sigma_{1}\right) \cdot r_{j k}\left(\tau_{2}\right)\right)\right. \\
& -C_{k i}\left(\sigma_{3}\right)\left(C_{j k}\left(\sigma_{2}\right) C_{k i}\left(\sigma_{3}\right)-V_{j k}\left(\sigma_{2}\right) \cdot V_{k i}\left(\sigma_{3}\right)\right)\left(V_{i j}\left(\sigma_{1}\right) \cdot r_{j k}\left(\tau_{2}\right)\right) \\
& +C_{j k}\left(\sigma_{2}\right)\left(V_{i j}\left(\sigma_{1}\right) \cdot V_{k i}\left(\sigma_{3}\right)\right)\left(V_{k i}\left(\sigma_{3}\right) \cdot r_{j k}\left(\tau_{2}\right)\right) \\
& \left.-C_{k i}\left(\sigma_{3}\right)\left(V_{i j}\left(\sigma_{1}\right) \cdot V_{j k}\left(\sigma_{2}\right)\right)\left(V_{k i}\left(\sigma_{3}\right) \cdot r_{j k}\left(\tau_{2}\right)\right)\right\} \text {, } \\
& P_{3}=7-2\left(C_{j k}\left(\sigma_{2}\right) C_{k i}\left(\sigma_{3}\right)-V_{j k}\left(\sigma_{2}\right) \cdot V_{k i}\left(\sigma_{3}\right)\right)^{2} \\
& +4\left(C_{i j}\left(\sigma_{1}\right) C_{j k}\left(\sigma_{2}\right)-V_{i j}\left(\sigma_{1}\right) \cdot V_{j k}\left(\sigma_{2}\right)\right)^{2}+4\left(C_{i j}\left(\sigma_{1}\right) C_{k i}\left(\sigma_{3}\right)-V_{i j}\left(\sigma_{1}\right) \cdot V_{k i}\left(\sigma_{3}\right)\right)^{2} \\
& +16\left\{-\left(C_{j k}\left(\sigma_{2}\right) C_{k i}\left(\sigma_{3}\right)-V_{j k}\left(\sigma_{2}\right) \cdot V_{k i}\left(\sigma_{3}\right)\right)\right. \\
& -\left(C_{i j}\left(\sigma_{1}\right) C_{j k}\left(\sigma_{2}\right)-V_{i j}\left(\sigma_{1}\right) \cdot V_{j k}\left(\sigma_{2}\right)\right)\left(C_{i j}\left(\sigma_{1}\right) C_{k i}\left(\sigma_{3}\right)-V_{i j}\left(\sigma_{1}\right) \cdot V_{k i}\left(\sigma_{3}\right)\right) \\
& +\left(C_{i j}\left(\sigma_{1}\right) C_{j k}\left(\sigma_{2}\right)-V_{i j}\left(\sigma_{1}\right) \cdot V_{j k}\left(\sigma_{2}\right)\right) \\
& +\left(C_{i j}\left(\sigma_{1}\right) C_{k i}\left(\sigma_{3}\right)-V_{i j}\left(\sigma_{1}\right) \cdot V_{k i}\left(\sigma_{3}\right)\right)\left(C_{j k}\left(\sigma_{2}\right) C_{k i}\left(\sigma_{3}\right)-V_{j k}\left(\sigma_{2}\right) \cdot V_{k i}\left(\sigma_{3}\right)\right) \\
& +\left(C_{i j}\left(\sigma_{1}\right) C_{k i}\left(\sigma_{3}\right)-V_{i j}\left(\sigma_{1}\right) \cdot V_{k i}\left(\sigma_{3}\right)\right) \\
& \left.+\left(C_{i j}\left(\sigma_{1}\right) C_{j k}\left(\sigma_{2}\right)-V_{i j}\left(\sigma_{1}\right) \cdot V_{j k}\left(\sigma_{2}\right)\right)\left(C_{j k}\left(\sigma_{2}\right) C_{k i}\left(\sigma_{3}\right)-V_{j k}\left(\sigma_{2}\right) \cdot V_{k i}\left(\sigma_{3}\right)\right)\right\}, \text { (A.4) } \\
& P_{4}=-4\left(V_{i j}\left(\sigma_{1}\right) \cdot r_{i j}\left(\tau_{1}\right)\right)\left(V_{i j}\left(\sigma_{1}\right) \cdot r_{i j}\left(\tau_{2}\right)\right)\left\{2+\left(C_{j k}\left(\sigma_{2}\right) C_{k i}\left(\sigma_{3}\right)-V_{j k}\left(\sigma_{2}\right) \cdot V_{k i}\left(\sigma_{3}\right)\right)^{2}\right\} \\
& +8\left(V_{j k}\left(\sigma_{2}\right) \cdot r_{i j}\left(\tau_{1}\right)\right)\left(V_{j k}\left(\sigma_{2}\right) \cdot r_{i j}\left(\tau_{2}\right)\right)\left\{1+\left(C_{i j}\left(\sigma_{1}\right) C_{k i}\left(\sigma_{3}\right)-V_{i j}\left(\sigma_{1}\right) \cdot V_{k i}\left(\sigma_{3}\right)\right)^{2}\right\}
\end{aligned}
$$




$$
\begin{aligned}
& +8\left(V_{k i}\left(\sigma_{3}\right) \cdot r_{i j}\left(\tau_{1}\right)\right)\left(V_{k i}\left(\sigma_{3}\right) \cdot r_{i j}\left(\tau_{2}\right)\right)\left\{1+\left(C_{i j}\left(\sigma_{1}\right) C_{j k}\left(\sigma_{2}\right)-V_{i j}\left(\sigma_{1}\right) \cdot V_{j k}\left(\sigma_{2}\right)\right)^{2}\right\} \\
& +4\left(V_{i j}\left(\sigma_{1}\right) \cdot V_{k i}\left(\sigma_{3}\right)\right)\left\{V_{i j}\left(\sigma_{1}\right) \cdot\left(r_{i j}\left(\tau_{1}\right)-r_{k i}\left(\tau_{1}\right)\right)\right\}\left\{V_{k i}\left(\sigma_{3}\right) \cdot\left(r_{j k}\left(\tau_{2}\right)-r_{i j}\left(\tau_{2}\right)\right)\right\} \\
& +4\left(V_{i j}\left(\sigma_{1}\right) \cdot V_{j k}\left(\sigma_{2}\right)-2 C_{i j}\left(\sigma_{1}\right) C_{j k}\left(\sigma_{2}\right)\right) \\
& \quad \times\left\{V_{j k}\left(\sigma_{2}\right) \cdot\left(r_{i j}\left(\tau_{1}\right)-r_{k i}\left(\tau_{1}\right)\right)\right\}\left\{V_{i j}\left(\sigma_{1}\right) \cdot\left(r_{j k}\left(\tau_{2}\right)-r_{i j}\left(\tau_{2}\right)\right)\right\} \\
& +4\left\{-C_{j k}\left(\sigma_{2}\right) C_{k i}\left(\sigma_{3}\right)+V_{j k}\left(\sigma_{2}\right) \cdot V_{k i}\left(\sigma_{3}\right)+2 C_{i j}\left(\sigma_{1}\right)^{2} C_{j k}\left(\sigma_{2}\right) C_{k i}\left(\sigma_{3}\right)\right. \\
& \left.+2\left(V_{i j}\left(\sigma_{1}\right) \cdot V_{j k}\left(\sigma_{2}\right)\right)\left(V_{i j}\left(\sigma_{1}\right) \cdot V_{k i}\left(\sigma_{3}\right)\right)-4 C_{i j}\left(\sigma_{1}\right) C_{j k}\left(\sigma_{2}\right)\left(V_{i j}\left(\sigma_{1}\right) \cdot V_{k i}\left(\sigma_{3}\right)\right)\right\} \\
& \quad \times\left\{V_{j k}\left(\sigma_{2}\right) \cdot\left(r_{i j}\left(\tau_{1}\right)-r_{k i}\left(\tau_{1}\right)\right)\right\}\left\{V_{k i}\left(\sigma_{3}\right) \cdot\left(r_{j k}\left(\tau_{2}\right)-r_{i j}\left(\tau_{2}\right)\right)\right\} \\
& +32\left\{-2\left(V_{j k}\left(\sigma_{2}\right) \cdot r_{j k}\left(\tau_{1}\right)\right)\left(V_{k i}\left(\sigma_{3}\right) \cdot r_{k i}\left(\tau_{2}\right)\right)-\left(V_{k i}\left(\sigma_{3}\right) \cdot r_{j k}\left(\tau_{1}\right)\right)\left(V_{j k}\left(\sigma_{2}\right) \cdot r_{k i}\left(\tau_{2}\right)\right)\right. \\
& +\left(C_{j k}\left(\sigma_{2}\right) C_{k i}\left(\sigma_{3}\right)-V_{j k}\left(\sigma_{2}\right) \cdot V_{k i}\left(\sigma_{3}\right)\right)\left(V_{i j}\left(\sigma_{1}\right) \cdot r_{j k}\left(\tau_{1}\right)\right)\left(V_{i j}\left(\sigma_{1}\right) \cdot r_{k i}\left(\tau_{2}\right)\right) \\
& -\left(C_{i j}\left(\sigma_{1}\right) C_{j k}\left(\sigma_{2}\right)-V_{i j}\left(\sigma_{1}\right) \cdot V_{j k}\left(\sigma_{2}\right)\right)\left(V_{k i}\left(\sigma_{3}\right) \cdot r_{j k}\left(\tau_{1}\right)\right)\left(V_{i j}\left(\sigma_{1}\right) \cdot r_{k i}\left(\tau_{2}\right)\right) \\
& \left.-\left(C_{i j}\left(\sigma_{1}\right) C_{k i}\left(\sigma_{3}\right)-V_{i j}\left(\sigma_{1}\right) \cdot V_{k i}\left(\sigma_{3}\right)\right)\left(V_{i j}\left(\sigma_{1}\right) \cdot r_{j k}\left(\tau_{1}\right)\right)\left(V_{j k}\left(\sigma_{2}\right) \cdot r_{k i}\left(\tau_{2}\right)\right)\right\} . \quad(\mathrm{A.5})
\end{aligned}
$$

\section{B Examples of evaluations of P's}

In this appendix, we present some examples of evaluations of $\tilde{\Gamma}_{(2)}$ from individual terms in (3.32). For each example below, we first write down the whole expression of the oneparticle irreducible, planar contractions, and then present the final form which fits into the form (3.33).

The first two terms in (3.32) involve the quartic vertices and contribute to $P_{q}$.

$$
\begin{aligned}
& \frac{1}{4} g^{2} \kappa \int d \tau\left\langle\operatorname{tr}\left[Y^{n}(\tau), Y^{m}(\tau)\right]\left[Y^{n}(\tau), Y^{m}(\tau)\right]\right\rangle_{1 P I, \text { planar }} \\
& =\frac{1}{2} g^{2} \kappa \int d \tau \sum_{i, j, k}\left(\left\langle Y_{i j}^{n}(\tau) Y_{j i}^{m}(\tau)\right\rangle_{0}\left\langle Y_{i k}^{n}(\tau) Y_{k i}^{m}(\tau)\right\rangle_{0}-\left\langle Y_{i j}^{n}(\tau) Y_{j i}^{n}(\tau)\right\rangle_{0}\left\langle Y_{i k}^{m}(\tau) Y_{k i}^{m}(\tau)\right\rangle_{0}\right) \\
& =\frac{g^{2}}{\kappa} \int d \tau_{1} d \tau_{2} d \sigma_{1} d \sigma_{2} d \sigma_{3} \sum_{i, j, k}\left\{-36-8 V_{j k}\left(\sigma_{2}\right)^{2}-8 V_{k i}\left(\sigma_{3}\right)^{2}-2 V_{j k}\left(\sigma_{2}\right)^{2} V_{k i}\left(\sigma_{3}\right)^{2}\right. \\
& \left.+2\left(V_{j k}\left(\sigma_{2}\right) \cdot V_{k i}\left(\sigma_{3}\right)\right)^{2}\right\}\left\{-\partial_{\sigma_{1}} \Delta_{i j}\left(\sigma_{1}, \tau_{1}, \tau_{2}\right)\right\} \Delta_{j k}\left(\sigma_{2}, \tau_{1}, \tau_{2}\right) \Delta_{k i}\left(\sigma_{3}, \tau_{1}, \tau_{2}\right), \\
& \frac{1}{2} g^{2} \kappa \int d \tau\left\langle\operatorname{tr}\left[\tilde{A}(\tau), Y^{m}(\tau)\right]\left[\tilde{A}(\tau), Y^{m}(\tau)\right]\right\rangle_{1 P I, \text { planar }} \\
& =g^{2} \kappa \int d \tau \sum_{i, j, k}\left(\left\langle\tilde{A}_{i j}(\tau) Y_{j i}^{m}(\tau)\right\rangle_{0}\left\langle\tilde{A}_{i k}(\tau) Y_{k i}^{m}(\tau)\right\rangle_{0}-\left\langle\tilde{A}_{i j}(\tau) \tilde{A}_{j i}(\tau)\right\rangle_{0}\left\langle Y_{i k}^{m}(\tau) Y_{k i}^{m}(\tau)\right\rangle_{0}\right. \\
& \left.+\left\langle\tilde{A}_{i j}(\tau) Y_{j i}^{m}(\tau)\right\rangle_{0}\left\langle Y_{j k}^{m}(\tau) \tilde{A}_{k j}(\tau)\right\rangle_{0}-\left\langle\tilde{A}_{i j}(\tau) Y_{j i}^{m}(\tau)\right\rangle_{0}\left\langle\tilde{A}_{j k}(\tau) Y_{k j}^{m}(\tau)\right\rangle_{0}\right)
\end{aligned}
$$




$$
\begin{aligned}
=\frac{g^{2}}{\kappa} \int d \tau_{1} d \tau_{2} d \sigma_{1} d \sigma_{2} d \sigma_{3} \sum_{i, j, k}\left\{-9-10 V_{j k}\left(\sigma_{2}\right)^{2}-10 V_{k i}\left(\sigma_{3}\right)^{2}\right. \\
\left.+12 C_{j k}\left(\sigma_{2}\right) C_{k i}\left(\sigma_{3}\right)\left(V_{j k}\left(\sigma_{2}\right) \cdot V_{k i}\left(\sigma_{3}\right)\right)-4 V_{j k}\left(\sigma_{2}\right)^{2} V_{k i}\left(\sigma_{3}\right)^{2}\right\} \\
\times\left\{-\partial_{\sigma_{1}} \Delta_{i j}\left(\sigma_{1}, \tau_{1}, \tau_{2}\right)\right\} \Delta_{j k}\left(\sigma_{2}, \tau_{1}, \tau_{2}\right) \Delta_{k i}\left(\sigma_{3}, \tau_{1}, \tau_{2}\right) .
\end{aligned}
$$

There are three terms which contain ghost fields. One of them involves two derivatives, thus contributes to the $P_{1}$ term:

$$
\begin{gathered}
-\frac{1}{2} g^{2} \kappa^{2} \int d \tau_{1} d \tau_{2}\left\langle\operatorname{tr} \partial_{\tau_{1}} \bar{c}\left(\tau_{1}\right)\left[\tilde{A}\left(\tau_{1}\right), c\left(\tau_{1}\right)\right] \operatorname{tr} \partial_{\tau_{2}} \bar{c}\left(\tau_{2}\right)\left[\tilde{A}\left(\tau_{2}\right), c\left(\tau_{2}\right)\right]\right\rangle_{1 P I, \text { planar }} \\
=-\frac{1}{2} g^{2} \kappa^{2} \int d \tau_{1} d \tau_{2} \sum_{i, j, k}\left(\left\langle\tilde{A}_{i j}\left(\tau_{1}\right) \tilde{A}_{j i}\left(\tau_{2}\right)\right\rangle_{0}\left\langle c_{j k}\left(\tau_{1}\right) \partial_{\tau_{2}} \bar{c}_{k j}\left(\tau_{2}\right)\right\rangle_{0}\left\langle\partial_{\tau_{1}} \bar{c}_{k i}\left(\tau_{1}\right) c_{i k}\left(\tau_{2}\right)\right\rangle_{0}\right. \\
\left.+\left\langle\tilde{A}_{i j}\left(\tau_{1}\right) \tilde{A}_{j i}\left(\tau_{2}\right)\right\rangle_{0}\left\langle\partial_{\tau_{1}} \bar{c}_{j k}\left(\tau_{1}\right) c_{k j}\left(\tau_{2}\right)\right\rangle_{0}\left\langle c_{k i}\left(\tau_{1}\right) \partial_{\tau_{2}} \bar{c}_{i k}\left(\tau_{2}\right)\right\rangle_{0}\right) \\
=\frac{g^{2}}{\kappa} \int d \tau_{1} d \tau_{2} d \sigma_{1} d \sigma_{2} d \sigma_{3} \sum_{i, j, k}\left(-\frac{1}{2}+V_{i j}\left(\sigma_{1}\right)^{2}-V_{j k}\left(\sigma_{2}\right)^{2}-V_{k i}\left(\sigma_{3}\right)^{2}\right) \\
\times\left\{\partial_{\tau_{1}} \partial_{\tau_{2}} \Delta_{i j}\left(\sigma_{1}, \tau_{1}, \tau_{2}\right)\right\} \Delta_{j k}\left(\sigma_{2}, \tau_{1}, \tau_{2}\right) \Delta_{k i}\left(\sigma_{3}, \tau_{1}, \tau_{2}\right)
\end{gathered}
$$

The term below has one derivative and contribute to $P_{2}$.

$$
\begin{aligned}
& i g^{2} \kappa^{2} \int d \tau_{1} d \tau_{2}\left\langle\operatorname{tr} \partial_{\tau_{1}} \bar{c}\left(\tau_{1}\right)\left[\tilde{A}\left(\tau_{1}\right), c\left(\tau_{1}\right)\right] \operatorname{tr}\left[B^{m}, \bar{c}\right]\left(\tau_{2}\right)\left[Y^{m}\left(\tau_{2}\right), c\left(\tau_{2}\right)\right]\right\rangle_{1 P I, \text { planar }} \\
& =i g^{2} \kappa^{2} \int d \tau_{1} d \tau_{2} \sum_{i, j, k}\left(\left\langle\tilde{A}_{i j}\left(\tau_{1}\right) Y_{j i}^{m}\left(\tau_{2}\right)\right\rangle_{0}\left\langle c_{j k}\left(\tau_{1}\right)\left[B^{m}, \bar{c}\right]_{k j}\left(\tau_{2}\right)\right\rangle_{0}\left\langle\partial_{\tau_{1}} \bar{c}_{k i}\left(\tau_{1}\right) c_{i k}\left(\tau_{2}\right)\right\rangle_{0}\right. \\
& \left.+\left\langle\tilde{A}_{i j}\left(\tau_{1}\right) Y_{j i}^{m}\left(\tau_{2}\right)\right\rangle_{0}\left\langle\partial_{\tau_{1}} \bar{c}_{j k}\left(\tau_{1}\right) c_{k j}\left(\tau_{2}\right)\right\rangle_{0}\left\langle c_{k i}\left(\tau_{1}\right)\left[B^{m}, \bar{c}\right]_{i k}\left(\tau_{2}\right)\right\rangle_{0}\right) \\
& =\frac{g^{2}}{\kappa} \int d \tau_{1} d \tau_{2} d \sigma_{1} d \sigma_{2} d \sigma_{3} \sum_{i, j, k}\left\{2 C_{i j}\left(\sigma_{1}\right)\left(V_{i j}\left(\sigma_{1}\right) \cdot r_{i j}\left(\tau_{2}\right)\right)\right. \\
& \left.-2 C_{j k}\left(\sigma_{2}\right)\left(V_{j k}\left(\sigma_{2}\right) \cdot r_{i j}\left(\tau_{2}\right)\right)-2 C_{k i}\left(\sigma_{3}\right)\left(V_{k i}\left(\sigma_{3}\right) \cdot r_{i j}\left(\tau_{2}\right)\right)\right\} \\
& \times\left\{\partial_{\tau_{1}} \Delta_{i j}\left(\sigma_{1}, \tau_{1}, \tau_{2}\right)\right\} \Delta_{j k}\left(\sigma_{2}, \tau_{1}, \tau_{2}\right) \Delta_{k i}\left(\sigma_{3}, \tau_{1}, \tau_{2}\right) .
\end{aligned}
$$

The last one involves no derivatives and contributes to both $P_{3}$ and $P_{4}$.

$$
\begin{aligned}
& \begin{array}{l}
\frac{1}{2} g^{2} \kappa^{2} \int d \tau_{1} d \tau_{2}\left\langle\operatorname{tr}\left[B^{n}, \bar{c}\right]\left(\tau_{1}\right)\left[Y^{n}\left(\tau_{1}\right), c\left(\tau_{1}\right)\right] \operatorname{tr}\left[B^{m}, \bar{c}\right]\left(\tau_{2}\right)\left[Y^{m}\left(\tau_{2}\right), c\left(\tau_{2}\right)\right]\right\rangle_{1 P I, \text { planar }} \\
=\frac{1}{2} g^{2} \kappa^{2} \int d \tau_{1} d \tau_{2} \sum_{i, j, k}\left(\left\langle Y_{i j}^{n}\left(\tau_{1}\right) Y_{j i}^{m}\left(\tau_{2}\right)\right\rangle_{0}\left\langle c_{j k}\left(\tau_{1}\right)\left[B^{m}, \bar{c}\right]_{k j}\left(\tau_{2}\right)\right\rangle_{0}\left\langle\left[B^{n}, \bar{c}\right]_{k i}\left(\tau_{1}\right) c_{i k}\left(\tau_{2}\right)\right\rangle_{0}\right. \\
\left.\quad+\left\langle Y_{i j}^{n}\left(\tau_{1}\right) Y_{j i}^{m}\left(\tau_{2}\right)\right\rangle_{0}\left\langle\left[B^{n}, \bar{c}\right]_{j k}\left(\tau_{1}\right) c_{k j}\left(\tau_{2}\right)\right\rangle_{0}\left\langle c_{k i}\left(\tau_{1}\right)\left[B^{m}, \bar{c}\right]_{i k}\left(\tau_{2}\right)\right\rangle_{0}\right)
\end{array} \\
& =\frac{g^{2}}{\kappa} \int d \tau_{1} d \tau_{2} d \sigma_{1} d \sigma_{2} d \sigma_{3} \sum_{i, j, k}\left\{-\frac{1}{2}\left(r_{i j}\left(\tau_{1}\right) \cdot r_{i j}\left(\tau_{2}\right)\right)+\left(V_{i j}\left(\sigma_{1}\right) \cdot r_{i j}\left(\tau_{1}\right)\right)\left(V_{i j}\left(\sigma_{1}\right) \cdot r_{i j}\left(\tau_{2}\right)\right)\right.
\end{aligned}
$$




$$
\begin{gathered}
\left.-\left(V_{j k}\left(\sigma_{2}\right) \cdot r_{i j}\left(\tau_{1}\right)\right)\left(V_{j k}\left(\sigma_{2}\right) \cdot r_{i j}\left(\tau_{2}\right)\right)-\left(V_{k i}\left(\sigma_{3}\right) \cdot r_{i j}\left(\tau_{1}\right)\right)\left(V_{k i}\left(\sigma_{3}\right) \cdot r_{i j}\left(\tau_{2}\right)\right)\right\} \\
\quad \times \Delta_{i j}\left(\sigma_{1}, \tau_{1}, \tau_{2}\right) \Delta_{j k}\left(\sigma_{2}, \tau_{1}, \tau_{2}\right) \Delta_{k i}\left(\sigma_{3}, \tau_{1}, \tau_{2}\right) .
\end{gathered}
$$

There are six terms which consist only of $\tilde{A}$ and $Y^{n}$. Each of these terms has twelve ways of one-particle irreducible, planar contractions and is much more complicated than the ghost terms. However, they can be arranged into the form (3.33) by similar transformations.

The final example is the terms with fermion fields. The three terms with fermion fields give contributions to $P_{1}, P_{2}, P_{3}$, and $P_{4}$.

$$
\begin{aligned}
& -\frac{1}{8} g^{2} \kappa^{2} \int d \tau_{1} d \tau_{2}\left\langle\operatorname{tr} \theta^{\alpha}\left(\tau_{1}\right)\left[\tilde{A}\left(\tau_{1}\right), \theta^{\alpha}\left(\tau_{1}\right)\right] \operatorname{tr} \theta^{\beta}\left(\tau_{2}\right)\left[\tilde{A}\left(\tau_{2}\right), \theta^{\beta}\left(\tau_{2}\right)\right]\right\rangle_{1 P I, \text { planar }} \\
& =-\frac{1}{2} g^{2} \kappa^{2} \int d \tau_{1} d \tau_{2} \sum_{i, j, k}\left\langle\tilde{A}_{i j}\left(\tau_{1}\right) \tilde{A}_{j i}\left(\tau_{2}\right)\right\rangle_{0}\left\langle\theta_{j k}^{\alpha}\left(\tau_{1}\right) \theta_{k j}^{\beta}\left(\tau_{2}\right)\right\rangle_{0}\left\langle\theta_{k i}^{\alpha}\left(\tau_{1}\right) \theta_{i k}^{\beta}\left(\tau_{2}\right)\right\rangle_{0} \\
& =\frac{g^{2}}{\kappa} \int d \tau_{1} d \tau_{2} d \sigma_{1} d \sigma_{2} d \sigma_{3} \sum_{i, j, k}\left[4 \left\{C_{j k}\left(\sigma_{2}\right) C_{k i}\left(\sigma_{3}\right)-V_{j k}\left(\sigma_{2}\right) \cdot V_{k i}\left(\sigma_{3}\right)\right.\right. \\
& +2 V_{i j}\left(\sigma_{1}\right)^{2} C_{j k}\left(\sigma_{2}\right) C_{k i}\left(\sigma_{3}\right)-2 V_{i j}\left(\sigma_{1}\right)^{2}\left(V_{j k}\left(\sigma_{2}\right) \cdot V_{k i}\left(\sigma_{3}\right)\right) \\
& -C_{i j}\left(\sigma_{1}\right) C_{k i}\left(\sigma_{3}\right)+V_{i j}\left(\sigma_{1}\right) \cdot V_{k i}\left(\sigma_{3}\right) \\
& -2 V_{j k}\left(\sigma_{2}\right)^{2} C_{i j}\left(\sigma_{1}\right) C_{k i}\left(\sigma_{3}\right)+2 V_{j k}\left(\sigma_{2}\right)^{2}\left(V_{i j}\left(\sigma_{1}\right) \cdot V_{k i}\left(\sigma_{3}\right)\right) \\
& -C_{i j}\left(\sigma_{1}\right) C_{j k}\left(\sigma_{2}\right)+V_{i j}\left(\sigma_{1}\right) \cdot V_{j k}\left(\sigma_{2}\right) \\
& \left.-2 V_{k i}\left(\sigma_{3}\right)^{2} C_{i j}\left(\sigma_{1}\right) C_{j k}\left(\sigma_{2}\right)+2 V_{k i}\left(\sigma_{3}\right)^{2}\left(V_{i j}\left(\sigma_{1}\right) \cdot V_{j k}\left(\sigma_{2}\right)\right)\right\} \\
& \times\left\{\partial_{\tau_{1}} \partial_{\tau_{2}} \Delta_{i j}\left(\sigma_{1}, \tau_{1}, \tau_{2}\right)\right\} \Delta_{j k}\left(\sigma_{2}, \tau_{1}, \tau_{2}\right) \Delta_{k i}\left(\sigma_{3}, \tau_{1}, \tau_{2}\right) \\
& +16\left\{C_{i j}\left(\sigma_{1}\right)\left(V_{j k}\left(\sigma_{2}\right) \cdot r_{j k}\left(\tau_{2}\right)\right)-C_{j k}\left(\sigma_{2}\right)\left(V_{i j}\left(\sigma_{1}\right) \cdot r_{j k}\left(\tau_{2}\right)\right)\right. \\
& \left.+2 C_{i j}\left(\sigma_{1}\right) V_{k i}\left(\sigma_{3}\right)^{2}\left(V_{j k}\left(\sigma_{2}\right) \cdot r_{j k}\left(\tau_{2}\right)\right)-2 C_{j k}\left(\sigma_{2}\right) V_{k i}\left(\sigma_{3}\right)^{2}\left(V_{i j}\left(\sigma_{1}\right) \cdot r_{j k}\left(\tau_{2}\right)\right)\right\} \\
& \times\left\{\partial_{\tau_{1}} \Delta_{i j}\left(\sigma_{1}, \tau_{1}, \tau_{2}\right)\right\} \Delta_{j k}\left(\sigma_{2}, \tau_{1}, \tau_{2}\right) \Delta_{k i}\left(\sigma_{3}, \tau_{1}, \tau_{2}\right) \\
& +4\left\{-C_{j k}\left(\sigma_{2}\right) C_{k i}\left(\sigma_{3}\right)-V_{j k}\left(\sigma_{2}\right) \cdot V_{k i}\left(\sigma_{3}\right)\right. \\
& -2 V_{i j}\left(\sigma_{1}\right)^{2} C_{j k}\left(\sigma_{2}\right) C_{k i}\left(\sigma_{3}\right)-2 V_{i j}\left(\sigma_{1}\right)^{2}\left(V_{j k}\left(\sigma_{2}\right) \cdot V_{k i}\left(\sigma_{3}\right)\right) \\
& +C_{i j}\left(\sigma_{1}\right) C_{k i}\left(\sigma_{3}\right)+V_{i j}\left(\sigma_{1}\right) \cdot V_{k i}\left(\sigma_{3}\right) \\
& +2 V_{j k}\left(\sigma_{2}\right)^{2} C_{i j}\left(\sigma_{1}\right) C_{k i}\left(\sigma_{3}\right)+2 V_{j k}\left(\sigma_{2}\right)^{2}\left(V_{i j}\left(\sigma_{1}\right) \cdot V_{k i}\left(\sigma_{3}\right)\right) \\
& +C_{i j}\left(\sigma_{1}\right) C_{j k}\left(\sigma_{2}\right)+V_{i j}\left(\sigma_{1}\right) \cdot V_{j k}\left(\sigma_{2}\right) \\
& \left.+2 V_{k i}\left(\sigma_{3}\right)^{2} C_{i j}\left(\sigma_{1}\right) C_{j k}\left(\sigma_{2}\right)+2 V_{k i}\left(\sigma_{3}\right)^{2}\left(V_{i j}\left(\sigma_{1}\right) \cdot V_{j k}\left(\sigma_{2}\right)\right)\right\}
\end{aligned}
$$




$$
\begin{gathered}
\times\left(r_{i j}\left(\tau_{1}\right) \cdot r_{i j}\left(\tau_{2}\right)\right) \Delta_{i j}\left(\sigma_{1}, \tau_{1}, \tau_{2}\right) \Delta_{j k}\left(\sigma_{2}, \tau_{1}, \tau_{2}\right) \Delta_{k i}\left(\sigma_{3}, \tau_{1}, \tau_{2}\right) \\
+8\left\{\left(V_{j k}\left(\sigma_{2}\right) \cdot r_{j k}\left(\tau_{1}\right)\right)\left(V_{k i}\left(\sigma_{3}\right) \cdot r_{k i}\left(\tau_{2}\right)\right)+\left(V_{k i}\left(\sigma_{3}\right) \cdot r_{j k}\left(\tau_{1}\right)\right)\left(V_{j k}\left(\sigma_{2}\right) \cdot r_{k i}\left(\tau_{2}\right)\right)\right. \\
+2 V_{i j}\left(\sigma_{1}\right)^{2}\left(V_{j k}\left(\sigma_{2}\right) \cdot r_{j k}\left(\tau_{1}\right)\right)\left(V_{k i}\left(\sigma_{3}\right) \cdot r_{k i}\left(\tau_{2}\right)\right) \\
\left.+2 V_{i j}\left(\sigma_{1}\right)^{2}\left(V_{k i}\left(\sigma_{3}\right) \cdot r_{j k}\left(\tau_{1}\right)\right)\left(V_{j k}\left(\sigma_{2}\right) \cdot r_{k i}\left(\tau_{2}\right)\right)\right\} \\
\left.\quad \times \Delta_{i j}\left(\sigma_{1}, \tau_{1}, \tau_{2}\right) \Delta_{j k}\left(\sigma_{2}, \tau_{1}, \tau_{2}\right) \Delta_{k i}\left(\sigma_{3}, \tau_{1}, \tau_{2}\right)\right] .
\end{gathered}
$$

\section{Formulas in calculating $\tilde{\Gamma}_{Y}$}

Here we supplement the derivation of $\tilde{\Gamma}_{Y}$ by providing some formulas. From the explicit form of the proper-time propagator (3.20), we have

$$
\begin{gathered}
\partial_{\tau_{1}} \Delta_{i j}\left(\sigma_{1}, \tau_{1}, \tau_{2}\right) \\
=\left[-\frac{V_{i j}\left(\sigma_{1}\right)}{C_{i j}\left(\sigma_{1}\right)} \cdot \frac{r_{i j}\left(\tau_{1}\right)+r_{i j}\left(\tau_{2}\right)}{2}-\frac{C_{i j}\left(\sigma_{1}\right)}{V_{i j}\left(\sigma_{1}\right)} \frac{\left|r_{i j}\left(\tau_{1}\right)-r_{i j}\left(\tau_{2}\right)\right|}{2}\right] \Delta_{i j}\left(\sigma_{1}, \tau_{1}, \tau_{2}\right), \\
\partial_{\tau_{1}} \partial_{\tau_{2}} \Delta_{i j}\left(\sigma_{1}, \tau_{1}, \tau_{2}\right) \\
=\left[-\frac{\tilde{v}_{i j}}{2} \frac{V_{i j}\left(\sigma_{1}\right)}{C_{i j}\left(\sigma_{1}\right)}+\frac{\tilde{v}_{i j}}{2} \frac{C_{i j}\left(\sigma_{1}\right)}{V_{i j}\left(\sigma_{1}\right)}+\left(\frac{V_{i j}\left(\sigma_{1}\right)}{C_{i j}\left(\sigma_{1}\right)} \cdot \frac{r_{i j}\left(\tau_{1}\right)+r_{i j}\left(\tau_{2}\right)}{2}\right)^{2}\right. \\
\frac{1}{2}\left(\partial_{\tau_{1}}^{2}+\partial_{\tau_{2}}^{2}\right) \Delta_{i j}\left(\sigma_{1}, \tau_{1}, \tau_{2}\right) \\
\left.=\left[-\frac{C_{i j}\left(\sigma_{1}\right)}{V_{i j}\left(\sigma_{1}\right)} \frac{r_{i j}\left(\tau_{1}\right)-r_{i j}\left(\tau_{2}\right)}{2}\right)^{2}\right] \Delta_{i j}\left(\sigma_{1}, \tau_{1}, \tau_{2}\right), \\
\quad-\frac{V_{i j}\left(\sigma_{1}\right)}{C_{i j}\left(\sigma_{1}\right)}-\frac{\tilde{v}_{i j}}{2} \frac{C_{i j}\left(\sigma_{1}\right)}{V_{i j}\left(\sigma_{1}\right)}+\left(\frac{V_{i j}\left(\sigma_{1}\right)}{C_{i j}\left(\sigma_{1}\right)} \cdot \frac{r_{i j}\left(\tau_{1}\right)+r_{i j}\left(\tau_{2}\right)}{2}\right)^{2} \\
\left.+\left(\frac{C_{i j}\left(\sigma_{1}\right)}{V_{i j}\left(\sigma_{1}\right)} \frac{r_{i j}\left(\tau_{1}\right)-r_{i j}\left(\tau_{2}\right)}{2}\right)^{2}\right] \Delta_{i j}\left(\sigma_{1}, \tau_{1}, \tau_{2}\right) .
\end{gathered}
$$

In general, $P_{Y}$ (3.47) has complicated dependence on $\tau_{1}$ and $\tau_{2}$. The dependence on $\{x \cdot \tilde{v}\}$

in proper-time propagators further complicates them. When we restrict to the case where $\{x \cdot \tilde{v}\}$ vanish, however, all we need is to notice the following equalities:

$$
\begin{gathered}
\int d \tau_{1} d \tau_{2}\left(\frac{\tau_{1}+\tau_{2}}{2}\right)^{2} \Delta_{i j}\left(\sigma_{1}, \tau_{1}, \tau_{2}\right) \Delta_{j k}\left(\sigma_{2}, \tau_{1}, \tau_{2}\right) \Delta_{k i}\left(\sigma_{3}, \tau_{1}, \tau_{2}\right) \\
=\int d \tau_{1} d \tau_{2} \frac{1}{2}\left(\frac{\tilde{v}_{i j} V_{i j}\left(\sigma_{1}\right)}{C_{i j}\left(\sigma_{1}\right)}+\frac{\tilde{v}_{j k} V_{j k}\left(\sigma_{2}\right)}{C_{j k}\left(\sigma_{2}\right)}+\frac{\tilde{v}_{k i} V_{k i}\left(\sigma_{3}\right)}{C_{k i}\left(\sigma_{3}\right)}\right)^{-1} \\
\times \Delta_{i j}\left(\sigma_{1}, \tau_{1}, \tau_{2}\right) \Delta_{j k}\left(\sigma_{2}, \tau_{1}, \tau_{2}\right) \Delta_{k i}\left(\sigma_{3}, \tau_{1}, \tau_{2}\right)
\end{gathered}
$$




$$
\begin{gathered}
\int d \tau_{1} d \tau_{2}\left(\frac{\tau_{1}-\tau_{2}}{2}\right)^{2} \Delta_{i j}\left(\sigma_{1}, \tau_{1}, \tau_{2}\right) \Delta_{j k}\left(\sigma_{2}, \tau_{1}, \tau_{2}\right) \Delta_{k i}\left(\sigma_{3}, \tau_{1}, \tau_{2}\right) \\
=\int d \tau_{1} d \tau_{2} \frac{1}{2}\left(\frac{\tilde{v}_{i j} C_{i j}\left(\sigma_{1}\right)}{V_{i j}\left(\sigma_{1}\right)}+\frac{\tilde{v}_{j k} C_{j k}\left(\sigma_{2}\right)}{V_{j k}\left(\sigma_{2}\right)}+\frac{\tilde{v}_{k i} C_{k i}\left(\sigma_{3}\right)}{V_{k i}\left(\sigma_{3}\right)}\right)^{-1} \\
\times \Delta_{i j}\left(\sigma_{1}, \tau_{1}, \tau_{2}\right) \Delta_{j k}\left(\sigma_{2}, \tau_{1}, \tau_{2}\right) \Delta_{k i}\left(\sigma_{3}, \tau_{1}, \tau_{2}\right) .
\end{gathered}
$$

\section{References}

[1] J. Polchinski, Phys. Rev. Lett. 75, (1995)4724.

[2] E. Witten, Nucl. Phys. B443 (1995) 85.

[3] T. Banks, W. Fischler, S. H. Shenker and L. Susskind, Phys. Rev. D55(1997)5112.

[4] M. Douglas, H. Ooguri and S. H. Shenker, Phys. Lett. B402(1997) 36; M. Douglas and H. Ooguri, hep-th/9710178.

[5] O. Ganor, R. Gopakumar and S. Ramgoolam, hep-th/9705188.

[6] M. Dine and A. Rajaraman, hep-th/9710174.

[7] E. Keski-Vakkuri and P. Kraus, hep-th/9712013. See also K. Becker and M. Becker, Phys. Rev. D57(1998)6464.

[8] K. Becker and M. Becker, Nucl. Phys. B506(1997)48.

[9] K. Becker, M. Becker, J. Polchinski and A. Tseytlin, Phys. Rev. D56(1997) 3174.

[10] P. C. Aichelburg and R. U. Sexl, Gen. Rel. Grav. 2 (1971) 303.

[11] R. Geroch and P. S. Jang, Jour. Math. Phys. 16(1975) 65.

[12] D. Kabat and W.Taylor, hep-th/9711078.

[13] L. Infeld and A. Schild, Rev. Mod. Phys. 21(1949) 408.

[14] L. Susskind, hep-th/9704080.

[15] T. Yoneya, Prog. Theor. Phys. 51(1974) 1907; Lett. Nuovo. Cim. 8(1973)951. 
[16] J. Scherk and J. Schwarz, Nucl. Phys. B81(1974)118.

[17] W. Wyss, Helv. Phys. Acta. 38(1965) 469.

S. Deser, Gen. Rel. Grav. 1(1970) 9.

[18] A. Jevicki and T. Yoneya, hep-th/9805069.

[19] M. Li and T. Yoneya, Phys. Rev. Lett. 78(1997) 1219.

[20] M. Fabbrichesi, G. Ferretti and R. Iengo, hep-th/9806018.

[21] W. Taylor IV and M. V. Raamsdonk, hep-th/9806066. 